\title{
Encoding Implicit Relation Requirements for Relation Extraction: A Joint Inference Approach
}

\author{
Liwei Chen ${ }^{\mathrm{a}}$, Yansong Feng ${ }^{\mathrm{a}, *}$, Songfang Huang ${ }^{\mathrm{b}}$, Bingfeng Luo ${ }^{\mathrm{a}}$, Dongyan Zhao ${ }^{\mathrm{a}}$ \\ ${ }^{a}$ Peking University, 128 Zhong Guan Cun North Street, Haidian, Beijing, China \\ ${ }^{b}$ IBM China Research Lab, Haidian, Beijing, China
}

\begin{abstract}
Relation extraction is the task of identifying predefined relationship between entities, and plays an essential role in information extraction, knowledge base construction, question answering and so on. Most existing relation extractors make predictions for each entity pair locally and individually, while ignoring implicit global clues available across different entity pairs and in the knowledge base, which often leads to conflicts among local predictions from different entity pairs. This paper proposes a joint inference framework that employs such global clues to resolve disagreements among local predictions. We exploit two kinds of clues to generate constraints which can capture the implicit type and cardinality requirements of a relation. Those constraints can be examined in either hard style or soft style, both of which can be effectively explored in an integer linear program formulation. Experimental results on both English and Chinese datasets show that our proposed framework can effectively utilize those two categories of global clues and resolve the disagreements among local predictions, thus improve various relation extractors when such clues are applicable to the datasets. Our experiments also indicate that the clues learnt automatically from existing knowledge bases perform comparably to or better than those refined by human.
\end{abstract}

Keywords: relation extraction, joint inference, knowledge base, integer linear programming

\footnotetext{
* Corresponding author

Email addresses: chenliwei@pku.edu.cn (Liwei Chen), fengyansong@pku.edu.cn (Yansong Feng), huangsf@cn. ibm. com (Songfang Huang), bf_luo@pku .edu.cn (Bingfeng Luo), zhaodongyan@pku.edu.cn (Dongyan Zhao)
} 


\section{Introduction}

Identifying predefined relationship between pairs of entities is crucial for many knowledge base related tasks, such as automatic knowledge base construction/population, factoid question answering, information extraction and so on, all of which have been popular topics in the field of artificial intelligence [1]. In the literature, the relation extraction task (RE) is usually investigated in a classification style, where relations are simply treated as isolated class labels, while their definitions or background information are sometimes ignored. Take the relation Capital as an example, we usually hold an agreement implicitly that this relation will expect a country as its subject and a city as object, and in most cases, a city can be the capital of only one country. In a knowledge base, such clues about a relation's requirements are usually defined as the domain and range of a relation, which are no doubt helpful for improving relation extraction. For instance, [2, 3, 4] all explicitly modeled the expected types of a relation's arguments with the help of a knowledge base's type taxonomy and obtained promising results for various information extraction tasks.

However, properly capturing and utilizing such typing clues are not trivial. One of the hurdles here is the lack of off-the-shelf resources and such clues often have to be coded by human experts. Many knowledge bases do not have a well-defined typing system, let alone fine-grained typing taxonomies with corresponding type recognizers, which are crucial to explicitly model the typing requirements for arguments of a relation, but rather expensive and time-consuming to collect. Similarly, the cardinality requirements of arguments, e.g., a person can have only one birthdate and a city can only be labeled as capital of only one country, should be considered as a strong indicator to eliminate wrong predictions, but has to be coded manually as well. Therefore, except expensive explicit treatment, it would be a better choice to implicitly mine such requirements from data. 
On the other hand, most previous relation extractors process each entity pair ${ }^{1}$ locally and individually, i.e., the extractor makes decisions solely based on the sentences containing the current entity pair and ignores other related pairs, therefore has difficulties to capture possible disagreements among different entity pairs. However, when looking at the output of a multi-class relation predictor globally, we can easily find possible incorrect predictions such as a university locates in two different cities, two different cities have been labeled as capital for one country, a country locates in a city and so on. Suppose we have two entity pairs, $<$ Richard Fuld, USA $>$ and $<$ USA, Washington $>$. The extractor predicts that the relation between the first pair is Nationality and the relation between the second one is LocationCity. It is easy to find that one of the two predictions should be incorrect, since the first prediction considers USA as a country but the second claims that USA locates in a city. We may also find such disagreements inside an entity pair, e.g., the extractor may predict a relation Capital for the second entity pair according to another instance, which obviously disagrees with the previous prediction LocationCity.

Resolving those disagreements mentioned above can be considered as a kind of constrained optimization tasks, e.g., optimizing the local predictions with global constraints among them, which can be captured by mining relation background related clues from the knowledge base. Based on those clues, we can discover the disagreements among the local predictions and generate constraints to resolve those conflicts. In this paper, we will address how to derive and exploit two categories of clues: the expected types and the cardinality requirements of a relation's arguments, to improve the performance of relation extraction. We propose to perform joint inference upon multiple local predictions by leveraging implicit clues that are encoded with relation specific requirements and can be learnt from existing knowledge bases. Specifically, the joint inference framework operates on the output of a sentence level relation extractor as input, derives different types of constraints from an existing KB to implicitly capture the expected type and cardinality requirements for a relation's arguments, and jointly resolve the disagreements among candidate predictions. We obtain 5 types

\footnotetext{
${ }^{1}$ we will use entity pair and entity tuple exchangeably in the rest of the paper
} 
of constraints in total, where three of them correspond to the type requirements and the rest ones relate to the cardinality requirements. We formalize this procedure as a constrained optimization problem, which can be solved by many optimization frameworks. The constraints we generated are also flexible to be utilized in either hard style or soft style. We use integer linear programming (ILP) as the solver and evaluate our framework on both English and Chinese datasets. The experimental results show that our framework can improve various relation extraction models, not only traditional feature based ones but also modern neural networks based. We find that the global clues can be learnt automatically from a knowledge base with better or comparable performance to those refined manually, which can be effectively investigated in either hard style or soft style formulations. Our framework can outperform the state-of-the-art approaches when such clues are applicable to the datasets.

In the rest of the paper, we first review related work in Section 2 , and in Section 3 , we describe our framework in detail. Experimental setup and results are discussed in Section 4 . We conclude this paper in Section 5

\section{Related Work}

The task of relation extraction can be divided into two major categories: sentencelevel (or mention-level) and entity pair level (or bag-level). The former predicts what relation a pair of entities may hold within the given sentence, and the latter focuses on identifying the relationship between an entity pair based on one or multiple sentences containing that entity pair. The entity pair level relation extraction task is more common in the literature, especially for the knowledge base construction related tasks, such as knowledge base population (KBP) [5] or automatically knowledge base construction (AKBC) [1].

Since traditional supervised relation extraction methods [6, 7, 8] require manual annotations and are often domain-specific, nowadays many efforts focus on open information extraction, which can extract hundreds of thousands of relations from large scale of web texts using semi-supervised or unsupervised methods [9, 10, 11, 12, 13, 14]. However, these relations are often not canonicalized, therefore are difficult to be mapped to 
an existing $\mathrm{KB}$.

Distant supervision (DS) is a semi-supervised relation extraction framework, which can automatically construct training data by aligning the triples in a KB to the sentences which contain their subjects and objects, and this learning paradigm has attracted much attention in information extraction tasks [15, 16, 2, 17, 18, 19, 20, 21]. DS approaches can predict canonicalized relations (predefined in a KB) for large amount of data and do not need much human involvement. Since the automatically generated training datasets in DS often contain noises, there are also research efforts focusing on reducing the noisy labels in the training data [22, 23], or utilizing human annotated data to improve the performance [24, 25]. Most of the above works put their emphasis on resolving or reducing the noises in the DS training data, but mostly focus on the extraction models themselves, i.e., improving the local extractors, while ignoring the inconsistencies among many local predictions.

As far as we know, few works have managed to take the relation specific requirements for arguments into account implicitly, and most existing works make predictions locally and individually, lacking in global considerations for inconsistency among local extractors. The MultiR system allows entity tuples to have more than one relations, but still predicts each entity tuple locally [18]. [19] propose a two-layer multi-instance multi-label (MIML) framework to capture the dependencies among relations. The first layer is a multi-class classifier making local predictions for single sentences, the output of which are aggregated by the second layer into the entity pair level. Their approach only captures relation dependencies, while we learn implicit relation backgrounds from knowledge bases, including argument type and cardinality requirements. [26] construct a set of relation topics, and integrate them into a relation detector for better relation predictions. [27 propose to use latent vectors to estimate the preferences between relations and entities. These can be considered as the latent type information about the relations' arguments, which is learnt from various data sources. In contrast, our approach can learn implicit clues from existing KBs, and jointly optimize local predictions among different entity tuples to capture both relation argument type clues and cardinality clues. [3] utilize relation cardinality to create negative samples for distant supervision while we use both implicit type clues and relation cardinality expectations 
to discover possible inconsistencies among local predictions. [2, 3, 4] propose to explicitly use the type information in distantly supervised relation extraction, which rely on existing typing resources and may have difficulties when the knowledge bases do not have fine types or sophisticated named entity taggers. In contrast, we try to implicitly mine both type and cardinality clues from $<$ subj, relation, obj $>$ triples without using fine typing tools or resources, which are then used to resolve the disagreements among local predictions.

In recent years, neural networks (NN) based models, such as PCNN [20], have been utilized in the relation extraction task, and the attention mechanism is also adopted to further reduce the noises within a sentence bag (that is, all the sentences containing an entity pair) [21]. [28] exploit class ties between relations within one entity tuple, and obtain promising results. However, those approaches still pay less attention to exploiting the possible dependencies between relations globally among all entity pairs. In contrast, our framework learns implicit clues from existing KBs, and jointly optimizes local predictions among different entity tuples to capture both relation argument type clues and cardinality clues. Specifically, this framework can lift various existing extractors, including traditional extractors and $\mathrm{NN}$ extractors.

There are also works which first represent relations and entities as embeddings in a $\mathrm{KB}$, and then utilize those embeddings to predict missing relations between any pair of entities in the KB [29, 30]. This task setup is different from ours, since we focus on extracting relations between entity pairs from the text resources, while they mainly make use of the structure information and descriptions of a KB to learn latent representations.

The idea of global optimization over local predictions has been proven to be helpful in other information extraction tasks. [31] and [32] use co-occurrence statistics among relations or events to jointly improve information extraction performances in ACE tasks, while we mine existing knowledge bases to collect global clues to solve local conflicts and find the optimal aggregation assignments, regarding existing knowledge facts. There are also works which encode general domain knowledge as first order logic rules in a topic model [33]. The main differences between their approach and our work are that our global clues can be collected from knowledge bases and our 
instantiated constraints are directly operated in an ILP model.

Most existing works in distantly supervised relation extraction focus on improving the performance locally, including designing sophisticated traditional or neural network models, incorporating explicit type information, reducing noisy instances in the training data, or utilizing extra human annotated data, etc. Different from previous works, we propose to implicitly exploiting the relation requirements to discover the disagreements among the unreliable local predictions, and formalize the procedure as a constrained optimization problem, which can resolve those disagreements and achieve a globally optimal assignment.

\section{The Framework}

Our framework takes a set of entity pairs and their supporting sentences as its input. We first train a preliminary sentence level extractor which can output confidence scores for its predictions, e.g., a maximum entropy or a neural network based model, and use this local extractor to produce local predictions. In order to implicitly capture the expected type and cardinality requirements for a relation's arguments, we derive two kinds of clues from an existing $\mathrm{KB}$, which are further utilized to discover the disagreements among local candidate predictions. Our objective is to maximize the overall confidence of all the selected predictions, as well as to minimize the inconsistencies among them. Figure 1 shows an overview of our proposed framework.

\subsection{Obtaining Candidate Relations}

Since we will focus on extracting relations predefined in a structured KB, we follow the distant supervision paradigm to collect our training data guided by this $\mathrm{KB}$, and train a local extractor accordingly, e.g., using an maximum entropy model, a neural network model, or other existing extractors. Given a sentence containing an entity pair $t$ (from a set of entity tuples $\mathcal{T}$ ), the model will output the confidence of this sentence representing certain relationship $r$ (from a predefined relation set $\mathcal{R}$ ) between the entity pair. Note that, due to the noisy nature of the training data, we admit that the resulting prediction is not fully reliable. But we still have a good chance to find the correct 


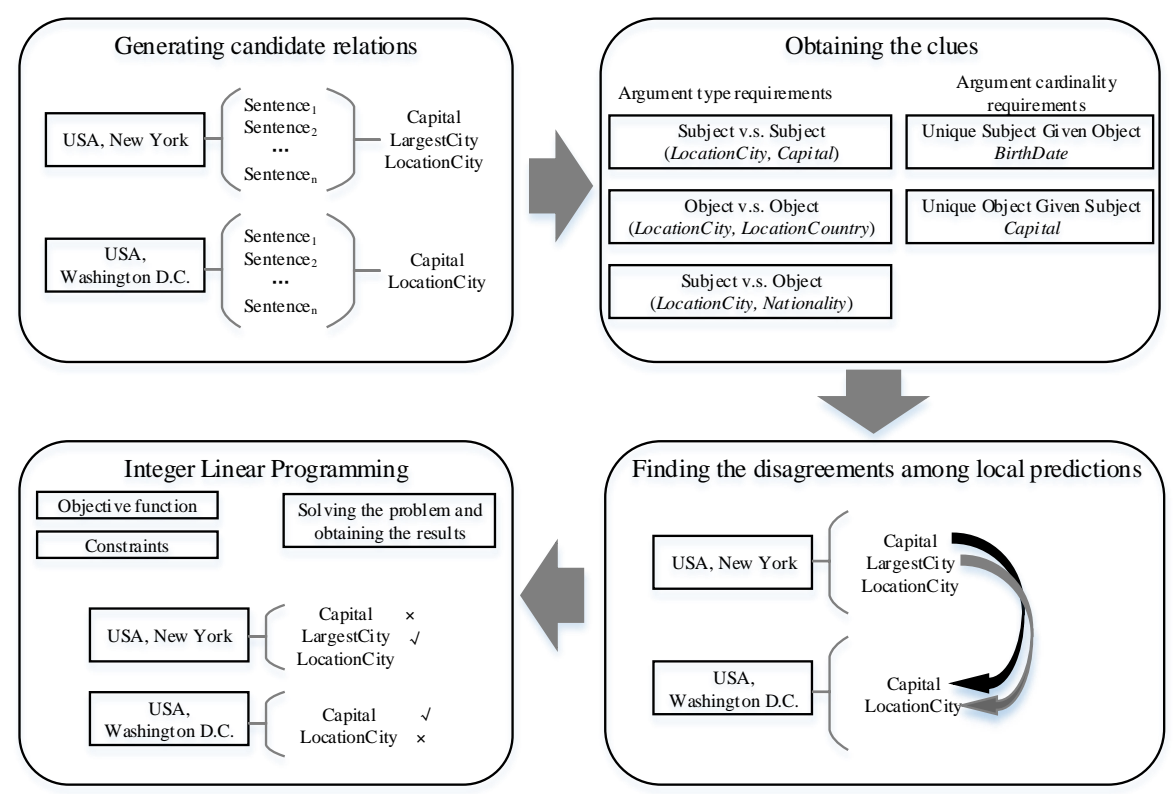

Figure 1: An overview illustration of our framework. The framework includes four main steps: generating the candidate relations, obtaining the clues, finding the disagreements among the local predictions, and using an integer linear programming framework to solve the problem and obtain the final results. 
prediction ranked in the second or third, we thus select top three predictions as the candidate relations for each entity pair in order to introduce more potentially correct output.

On the other hand, we should discard the predictions whose confidences are too low to be true. We set up a threshold of 0.1. For an entity tuple $t$, we obtain its candidate relation set by combining the candidate relations of all its sentence-level mentions, and represent it as $R^{t}$. For a candidate relation $r \in R^{t}$ and a tuple $t$, we define $M_{t}^{r}$ as all $t$ 's sentence-level mentions whose candidate relations contain $r$. Now the confidence score of a relation $r \in R^{t}$ being assigned to tuple $t$ can be calculated as:

$$
\operatorname{conf}(t, r)=\sum_{m \in M_{t}^{r}} \operatorname{score}(m, r)
$$

where $\operatorname{score}(m, r)$ is the confidence of a mention $m$ representing relation $r$ output by our preliminary extractor.

Traditionally, both lexical features and syntactic features have been investigated in the relation extraction task. Lexical features are the word chains between the subjects and objects in the sentences, which are usually too specific to frequently appear in the test data. For instance, a long lexical feature "PERSON was an American politician who was born on August 19, 1946 in LOCATION" extracted from the training set may be unlikely to exist in any sentence of the testing set, thus might be useless in predicting the relation BirthPlace in the future. On the other hand, the reliability of syntactic features depends heavily on the quality of dependency parsing tools, which may limit the usage of such kind of features in the languages which do not have high quality parsing toolkits.

Generally speaking, we expect more potentially correct relations to be put into the candidate relation set for further consideration, i.e., we expect recall-oriented preliminary local extractors. So, in addition to lexical and syntactic features, traditional feature based extractors can benefit from incorporating n-gram features, which are considered as more ambiguous and bring higher recall. In the neural network category, the NN extractors [20, 21] take advantage of word embeddings and convolutional architectures to naturally support recalling more potentially correct results. 


\subsection{Disagreements among the Candidates}

The candidate relations we obtained from local extractors inevitably include incorrect predictions, since they are often predicted individually, e.g., within one entity pair. One way to identify those incorrect predictions is to find any disagreements among local predictions, which could be resolved by discarding the local predictions that lead to the disagreements. This can help us to obtain more accurate predictions, with more global coherence. Therefore, our clues should be in a form of A-and-B-should-nothappen-together. Theoretically, our framework can deal with any sort of clues in such a form. In this paper, we will discuss two kinds of them in detail.

As discussed earlier, we will exploit from the knowledge base two categories of clues that implicitly capture relations' backgrounds: their expected argument types and argument cardinalities, based on which we can discover two categories of disagreements among the candidate predictions, summarized as argument type inconsistencies and violations of arguments' uniqueness, which have been rarely investigated before. Next, we will discuss them in detail, and describe how to learn the clues from a KB afterwards.

\subsubsection{Implicit Argument Types Inconsistencies:}

Generally, the argument types of correct predictions should be consistent with each other. Given a relation, its arguments sometimes are required to be certain types of entities. If the local predictions among different entity tuples require the same entity to belong to different and contradictory types, we call this situation as argument type inconsistency. Take $<$ USA, New York $>$ and $<$ USA, Washington D.C. $>$ as an example. In Figure $2<$ USA, New York $>$ has a candidate relation LargestCity which restricts USA to be either countries or states, while $<$ USA, Washington D.C. $>$ has a prediction LocationCity which requires an organization as its subject. These two local predictions lead to a disagreement in terms of USA's type because the latter expects USA to be an organization located in a city, which warns that at least one of the two candidate relations is incorrect.

Besides the disagreement between the subjects of two candidate relations, from Figure 2, we can observe two more situations: the first one is that the objects of the 


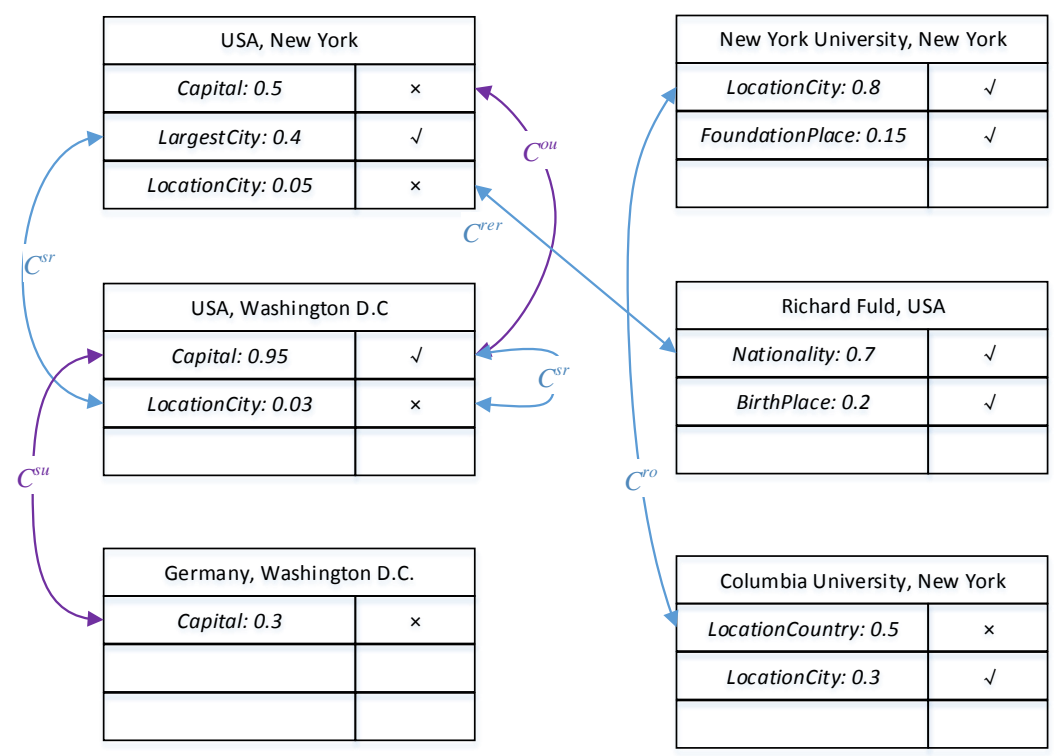

Figure 2: Different types of disagreements we investigate among the candidate relations. Each arrow link two local relations that conflict with each other. The color of an arrow indicates the type of the clue we use to discover the disagreement, and the caption of an arrow shows its subtypes. 
two candidate relations are inconsistent with each other, for example $<$ New York University, New York $>$ with the prediction LocationCity and $<$ Columbia University, New York $>$ with the prediction LocationCountry. The second one is that the subject of one candidate relation does not agree with another prediction's object, for example $<$ Richard Fuld, USA $>$ with the prediction Nationality and $<$ USA, New York $>$ with the prediction LocationCity. Although we have not assigned explicit types to these entities, we can still exploit the inconsistencies implicitly with the help of shared entities. Note that the implicit argument typing clues here mean whether two relations can share arguments, but not enumerate what types explicitly their arguments should have.

Formally, these problematic relation pairs can be divided into the following three subcategories. We represent the relation pairs $\left(r_{i}, r_{j}\right)$ that are inconsistent in terms of subjects as $\mathcal{C}^{s r}$, the relations pairs that are inconsistent in terms of objects as $\mathcal{C}^{\text {ro }}$, the relation pairs that are inconsistent in terms of one's subject and the other one's object as $\mathcal{C}^{\text {rer }}$.

It is worth mentioning that disagreements inside an entity tuple are also considered here. For instance, an entity tuple <USA, Washington D.C. $>$ in Figure 2 has two candidate relations, Capital and LocationCity. These two predictions are inconsistent with each other with respect to the type of USA. They implicitly consider USA as “country" and "organization", respectively.

\subsubsection{Violations of Arguments' Uniqueness:}

The second kind of disagreement regards the argument cardinality requirements for a relation. Given a subject, some relations should have unique objects. For example, in Figure 2, given USA as the subject of the relation Capital, we can only accept one possible object, because there is great chance that a country only have one capital. On the other hand, given Washington D.C. as the object of the relation Capital, we can only accept one subject, since usually a city can only be the capital of one country or state. If these are violated in the candidates, we could know that there may be some incorrect predictions. We represent the relations expecting unique objects as $\mathcal{C}^{o u}$, and the relations expecting unique subjects as $\mathcal{C}^{s u}$. 


\subsubsection{Other Types of Clues}

In addition to the two types of clues discussed above, there could be some other types of clues, in the form of A-and-B-should-not-happen-together, applicable to this task. For example, one may design a kind of clues with respect to the numerical values of different relations' arguments, e.g., one person's BirthDate should be earlier than his DeathDate, which can be easily transformed to a constraint regarding whether two local predictions can be correct at the same time.

Such type of clues are surely useful if they are applicable to the datasets. However, there are few such cases in our current datasets, we will leave further investigations as an interesting direction for future work.

\subsection{Obtaining the Global Clues}

Now, the issue is how to obtain the clues used in the previous subsection. That is, how we determine which relations expect certain types of subjects, which relations expect certain types of objects, etc. These knowledge can be definitely coded by human, or learnt from a KB.

Most existing knowledge bases represent their knowledge facts in the form of $(<$ subject, relation, object $>)$ triple, which can be seen as relational facts between entity tuples. Usually the triples in a KB are carefully defined by experts. It is rare to find inconsistencies among the triples in the knowledge base. The clues are therefore learnt from KBs, and further refined manually if needed.

Given two relations $r_{1}$ and $r_{2}$, we query the KB for all tuples bearing the relation $r_{1}$ or $r_{2}$. We use $S_{i}$ and $O_{i}$ to represent $r_{i}$ 's $(i \in\{1,2\})$ subject set and object set, respectively. We adopt a modified Kulczynski similarity coefficient $(\mathcal{K})[34]$ to estimate the dependency between the argument sets of two relations:

$$
\mathcal{K}(A, B)=\log \frac{1}{2}\left(\frac{\operatorname{count}(A \cap B)}{\operatorname{count}(A)}+\frac{\operatorname{count}(A \cap B)}{\operatorname{count}(B)}\right)
$$

where count $(A \cap B)$ is number of the entities in both $A$ and $B, \operatorname{count}(A)$ and $\operatorname{count}(B)$ are the numbers of the entities in $A$ and $B$, respectively. For any pair of relations from $\mathcal{R} \times \mathcal{R}$, we calculate four scores: $\mathcal{K}\left(S_{1}, S_{2}\right), \mathcal{K}\left(O_{1}, O_{2}\right), \mathcal{K}\left(S_{1}, O_{2}\right)$ and $\mathcal{K}\left(S_{2}, O_{1}\right)$. To make more stable estimations, we set up a threshold $\kappa$ for $\mathcal{K}()$. If $\mathcal{K}\left(S_{1}, S_{2}\right)$ is 
lower than the threshold, it means there is a good chance that $r_{1}$ and $r_{2}$ cannot share a subject, and we should generate a clue for them. Otherwise, we will consider that $r_{1}$ and $r_{2}$ can share a subject. Things are similar for the other three scores. The threshold is set to -3 in this paper.

We can also learn the uniqueness of arguments for relations. For each pre-defined relation in $\mathcal{R}$, we collect all the triples containing this relation, and count the portion of the triples which only have one object for each subject, and the portion of the triples which only have one subject for each object. The relations whose portions are higher than the threshold will be considered to have unique argument values. This threshold is set to 0.8 in this paper.

One issue for the learning strategy is when a relation has only a small amount of instances in the KB, the learnt clues about that relation can be inevitably noisy, which may bring errors to the final predictions. Here, we should take different application scenarios into account, to balance the human crafted clues and the automatically obtained ones. For example, during the initial stage of knowledge base population, i.e., when the KB is still small, we can first utilize manually crafted clues for new relations and existing relations with only a small number of instances. As we accumulate more and more relation instances and populate them into the $\mathrm{KB}$, we can move onto the automatically learnt clues.

\subsection{Integer Linear Program Formulation}

As discussed above, given a set of entity pairs and their candidate relations output by a preliminary extractor, our goal is to find an optimal configuration for all those entities pairs jointly, solving the disagreements among those candidate predictions and maximizing the overall confidence of the selected predictions. It has been proven that this problem is the reduction from an NP-complete problem, minimum vertex cover, which means it is an NP-hard problem ${ }^{2}$ Many optimization models can be used to obtain the approximate solutions. In this paper, we propose to solve the problem by

\footnotetext{
${ }^{2}$ https://en.wikipedia.org/wiki/Integer_programming
} 
using an ILP tool, IBM ILOG Cplex

\subsubsection{Objective Function}

For each tuple $t$ and one of its candidate relations $r$, we define a binary decision variable $d_{t}^{r} \in\{0,1\}$, indicating whether the candidate relation $r$ is selected by the solver. Our objective is to maximize the total confidence of all the selected candidates, and the objective function can be written as:

$$
\max \sum_{t \in \mathcal{T}, r \in R^{t}}\left(\operatorname{conf}(t, r)+\max _{\mathrm{m} \in M_{t}^{r}} \operatorname{score}(m, r)\right) d_{t}^{r}
$$

where $\operatorname{conf}(t, r)$ is the confidence of the tuple $t$ bearing the candidate relation $r, M_{t}^{r}$ is the set of $t$ 's sentence-level mentions whose candidate relations contain $r$. The first component is the original confidence scores of all the selected candidates, and the second one is the maximal mention-level confidence scores of all the selected candidates. The latter is designed to encourage the model to select the candidates with higher individual mention-level confidence scores, which, to some extent, coincides with the distant supervision assumption. For example, in an entity pair $t, 5$ mentions are predicted as $r_{1}$ with 0.2 confidence score by the local extractor, and another mention is predicted as $r_{2}$ with a confidence 0.9 , we would like to encourage the model to select the latter.

\subsubsection{Constraint Generation}

Now we formulate the clues we have collected into constraints to help resolve the disagreements among the candidate relations. Note that the formulation can be in either hard style or soft style.

The hard-style formulation will generate hard constraints based on those clues, which means if there are two predictions violating the constraints, we will have to discard the one which may result in a smaller objective, e.g., the one with lower confidence. For example, given two predictions which indicate that a country is also an organization locating in a city, there is a good chance that one of the predictions is incorrect and should be eliminated.

\footnotetext{
${ }^{3}$ Www.cplex.com
} 
On the other hand, the soft-style formulation allows a constraint to be violated, to some extent, with a continuous value as a kind of penalty. This is designed under the observation that some of the clues we have generated can be violated in certain situations. For example, in most cases an actor will not be a politician, but Arnold Schwarzenegger is both an actor and a politician. Thus, allowing the constraints to be violated with certain penalty can deal with such rare situations, and potentially avoid eliminating true positive samples according to one strict hard constraint. Intuitively, one should expect a higher penalty for very rare situations, and a smaller penalty for a constraint with several exceptions.

\subsubsection{Hard Style Formulation}

Here we describe how to formally take into consideration the two kinds of disagreements, i.e., implicit argument types inconsistencies and violations of arguments' uniqueness, in a hard style. For the sake of clarity, we describe the constraints derived from each scenario of these two disagreements separately.

The subject-relation constraints avoid the disagreements between the predictions of two tuples sharing a subject. These constraints can be represented as:

$$
\begin{array}{r}
d_{t_{i}}^{r^{t_{i}}}+d_{t_{j}}^{r_{j}} \leq 1 \\
\forall t_{i}, t_{j}: \operatorname{subj}\left(t_{i}\right)=\operatorname{subj}\left(t_{j}\right) \wedge\left(r^{t_{i}}, r^{t_{j}}\right) \in \mathcal{C}^{s r}
\end{array}
$$

where $t_{i}$ and $t_{j}$ are two tuples in $\mathcal{T}, \operatorname{subj}\left(t_{i}\right)$ is the subject of $t_{i}, r^{t_{i}}$ is a candidate relation of $t_{i}, r^{t_{j}}$ is a candidate relation of $t_{j}$.

The object-relation constraints avoid the inconsistencies between the predictions of two tuples sharing an object. Formally we add the following constraints:

$$
\begin{array}{r}
d_{t_{i}}^{r^{t_{i}}}+d_{t_{j}}^{r^{t_{j}}} \leq 1 \\
\forall t_{i}, t_{j}: o b j\left(t_{i}\right)=o b j\left(t_{j}\right) \wedge\left(r^{t_{i}}, r^{t_{j}}\right) \in \mathcal{C}^{r o}
\end{array}
$$

where $t_{i} \in \mathcal{T}$ and $t_{j} \in \mathcal{T}$ are two tuples, $\operatorname{obj}\left(t_{i}\right)$ is the object of $t_{i}$.

The relation-entity-relation constraints ensure that if an entity works as subject and object in two tuples $t_{i}$ and $t_{j}$, respectively, their relations should agree with each other. 
The constraints can be designed as:

$$
\begin{array}{r}
d_{t_{i}}^{r^{t_{i}}}+d_{t_{j}}^{r^{t_{j}}} \leq 1 \\
\forall t_{i}, t_{j}: \operatorname{obj}\left(t_{i}\right)=\operatorname{subj}\left(t_{j}\right) \wedge\left(r^{t_{i}}, r^{t_{j}}\right) \in \mathcal{C}^{r e r}
\end{array}
$$

The object uniqueness constraints ensure that the relations requiring unique objects do not bear more than one object given a subject.

$$
\begin{array}{r}
\sum_{t \in \operatorname{Tuple}(r), \operatorname{subj}(t)=e} d_{t}^{r} \leq 1 \\
\forall e, r: r \in \mathcal{C}^{o u}
\end{array}
$$

where $e$ is an entity, Tuple $(r)$ are the tuples whose candidate relations contain $r$.

Similarly, the subject uniqueness constraints ensure that given an object, the relations expecting unique subjects do not bear more than one subject.

$$
\begin{array}{r}
\sum_{t \in T u p l e(r), o b j(t)=e} d_{t}^{r} \leq 1 \\
\forall e, r: r \in \mathcal{C}^{s u}
\end{array}
$$

\subsubsection{Soft Style Formulation}

To utilize the constraints in a soft style, we give each constraint a non-negative penalty of violating it. The higher the penalty is, the more confident we are that the corresponding constraint should not be violated. An extreme case is that the constraint has a penalty of infinite, which means this constraint should not be violated at all, working as a hard constraint. Again, our aim is to find an optimal solution which maximizes the objective function (Equation 3) as defined in Section 3.4.1 and minimizes the penalty of violating the constraints at the same time.

We then rewrite the objective function (Equation 3 ) to accommodate constraints in a soft style. For each hard constraint $d_{t_{i}}^{r^{t_{i}}}+d_{t_{j}}^{r^{t_{j}}} \leq 1$ discussed in Section 3.4.3. we define a new decision variable, $d_{t_{i}, t_{j}}^{r^{t_{i}}, r_{j}^{t_{j}}}$, which will be 1 if the constraint is violated (that is, when both $d_{t_{i}}^{r_{i}}$ and $d_{t_{j}}^{r^{t_{j}}}$ are 1), and 0 otherwise. Note that each $d_{t_{i}, t_{j}}^{r^{t_{i}}, r_{j}^{t_{j}}}$ is still a binary decision variable, but each one will be associated with a continuous penalty of violating the corresponding constraint. However, for the constraints generated from 
the second category of clues (violations of argument uniqueness), it is difficult to fit them linearly into the objective function ${ }^{4}$

Therefore, in this paper, we only transform the constraints from implicit argument types inconsistencies into soft constraints. Starting from Equation 3 , we take into account the penalty values of violating the soft constraints, and formulate the total penalty as a new component of the objective, which can be defined as:

$$
\begin{aligned}
\max \left(\sum_{t \in \mathcal{T}, r \in R^{t}}(\operatorname{conf}(t, r)+\right. & \left.\left.\max _{\mathrm{m} \in M_{t}^{r}} \operatorname{score}(m, r)\right) d_{t}^{r}-\sum d_{t_{i}, t_{j}}^{r^{t_{i}}, r^{t_{j}}} p\left(r^{t_{i}}, r^{t_{j}}\right)\right) \\
& \forall t_{i}, t_{j}: \operatorname{subj}\left(t_{i}\right)=\operatorname{subj}\left(t_{j}\right) \wedge\left(r^{t_{i}}, r^{t_{j}}\right) \in \mathcal{C}^{s r} \\
& \forall t_{i}, t_{j}: \operatorname{obj}\left(t_{i}\right)=\operatorname{obj}\left(t_{j}\right) \wedge\left(r^{t_{i}}, r^{t_{j}}\right) \in \mathcal{C}^{r o} \\
& \forall t_{i}, t_{j}: \operatorname{obj}\left(t_{i}\right)=\operatorname{subj}\left(t_{j}\right) \wedge\left(r^{t_{i}}, r^{t_{j}}\right) \in \mathcal{C}^{r e r}
\end{aligned}
$$

The penalty values $p\left(r^{t_{i}}, r^{t_{j}}\right)$ of violating the constraints should be calculated based on the clues from which they are generated. However, it is impossible to manually assign proper penalty values for each of those clues, so we stick on the automatically generated clues for the soft constraints. There could be many ways to automatically obtain the penalty values, and one straightforward method is to use the $\mathcal{K}$ scores of the clues from which those constraints are generated:

$$
p\left(r^{t_{i}}, r^{t_{j}}\right)=-\alpha \mathcal{K}\left(r^{t_{i}}, r^{t_{j}}\right)
$$

where the parameter $\alpha$ adjusts the weights of the penalty of violating the constraints, and $\mathcal{K}\left(r^{t_{i}}, r^{t_{j}}\right)$ is calculated based on the argument sets of the two relations as described in Section 3.3 . Since the $\mathcal{K}$ scores are negative and the penalty values are usually considered as positive, we thus add a minus sign in the equation.

In order to restrict the decision variable $d_{t_{i}, t_{j}}^{r^{t_{i}}, r^{t_{j}}}$ to be 1 only when both $d_{t_{i}}^{r^{t_{i}}}$ and

\footnotetext{
${ }^{4}$ For a constraint like $\sum_{t \in \text { Tuple }(r), o b j(t)=e} d_{t}^{r} \leq 1$, it will be violated when more than one $d_{t}^{r}$ equal to 1 .

We need a decision variable similar with $d_{t_{i}, t_{j}}^{r^{t_{i}}, r^{t_{j}}}$ to represent the violation of the constraint. However, it is impossible to use a linear combination of several linear constraints to make the decision variable act as we expect.
} 
$d_{t_{j}}^{r^{t_{j}}}$ are 1 , we need to add the following new constraints:

$$
\begin{array}{r}
d_{t_{i}, t_{j}}^{r^{t_{i}}, r_{j}^{t_{j}}} \leq d_{t_{i}}^{r^{t_{i}}} \\
d_{t_{i}, t_{j}}^{r^{t_{i}}, r^{t_{j}}} \leq d_{t_{j}}^{r^{r_{j}}} \\
d_{t_{i}}^{r^{t_{i}}}+d_{t_{j}}^{r^{t_{j}}} \leq d_{t_{i}, t_{j}}^{r^{t_{i}}, r^{t_{j}}}+1 \\
\forall t_{i}, t_{j}: \operatorname{subj}\left(t_{i}\right)=\operatorname{subj}\left(t_{j}\right) \wedge\left(r^{t_{i}}, r^{t_{j}}\right) \in \mathcal{C}^{s r} \\
\forall t_{i}, t_{j}: \operatorname{obj}\left(t_{i}\right)=\operatorname{obj}\left(t_{j}\right) \wedge\left(r^{t_{i}}, r^{t_{j}}\right) \in \mathcal{C}^{r o} \\
\forall t_{i}, t_{j}: \operatorname{obj}\left(t_{i}\right)=\operatorname{subj}\left(t_{j}\right) \wedge\left(r^{t_{i}}, r^{t_{j}}\right) \in \mathcal{C}^{r e r}
\end{array}
$$

No matter how we formalize those constraints, hard or soft, the ILP based joint optimization framework helps us combine the confidence from local extractors and the implicit relation background encoded in the global consistencies among entity tuples together. After the optimization problem is solved, we will obtain a full configuration per each candidate relation, which will help us eliminate incorrect candidates.

\section{Experiments}

We will compare our proposed framework with both traditional and neural networks based state-of-the-art relation extractors on different datasets in different languages. Specifically, these experiments are designed to address the following questions: (a) whether our proposed framework can effectively handle the global inconsistency among local predictions? (b) whether our framework can work with automatically obtained clues, except manually designed clues? (c) how differently the framework performs with hard-style or soft-style constraints?

Next, we will introduce the datasets, describe the baseline models used for comparisons, and present the results in detail.

\subsection{Datasets}

We evaluate our approach on three datasets, including two English datasets and one Chinese dataset.

The first English dataset, Riedel's dataset, is the one used in [35, 18, 19], with the same training/test split as previous works. It uses Freebase as the knowledge base, 
covering 52 Freebase relations in total, and the New York Time corpus [36] as the text corpus, including about 281,000 entity tuples, 552,000 sentences in the training set, about 96,000 entity tuples, and 172,000 sentences in the testing set.

We construct another English dataset, the DBpedia dataset, by mapping the triples in DBpedia [37] to the sentences in the New York Time corpus. We map 51 different relations to the corpus and result in about 134,000 sentences with 50,000 entity tuples for training, and 53,000 sentences with 30,000 entity tuples for testing.

For the Chinese dataset, we derive knowledge facts and construct a Chinese KB from the Infoboxes of HudongBaike $\AA^{5}$ one of the largest Chinese online encyclopedias. We collect four national economic newspapers in 2009 as the text corpus. 28 different relations are mapped to the corpus, resulting in 60,000 entity tuples, 120,000 sentences for training, and 40,000 tuples, 83,000 sentences for testing.

We obtain two kinds of global clues, regarding implicit argument types inconsistencies and violations of arguments' uniqueness, respectively, for all three datasets. Each clue in the first category is a pair of relations, e.g., < country, nationality $>$ is a clue from $\mathcal{C}^{s r}$, which means it is unlikely that the two relations can share subjects. Each clue in the second category is a single relation who requires unique argument values. For example, capital is a relation from $\mathcal{C}^{o u}$, indicating that we can only accept one object given a specific subject for relation capital.

The Riedel's dataset is produced from a much earlier version of Freebase which we cannot access now, thus can not obtain any clues automatically. We therefore manually collect clues for this dataset only. The numbers of the clues manually and automatically obtained for those datasets are listed in Table 1 . We list the manually obtained clues for the DBpedia and Chinese datasets in the Appendix.

As for the number of the generated constraints on each dataset, if we consider all the predefined relations as candidates, there would be a huge number of constraints which only depend on the number of entity pairs and what clues we have. However, in practice we could select candidates from the top predictions of the local extractors as discussed in Section 3.1, thus the number of the constraints will also be related to the

\footnotetext{
${ }^{5}$ www.baike.com
} 
Table 1: The number of the clues on the three datasets.

\begin{tabular}{l|cc|cc}
\hline Dataset & \multicolumn{2}{|c|}{ Manual Clues } & \multicolumn{2}{c}{$\begin{array}{c}\text { Automated Clues } \\
\end{array}$} \\
& The 1st Category & The 2nd Category & The 1st Category & The 2nd Category \\
\hline Riedel's & 58 & 11 & - & - \\
DBpedia & 65 & 8 & 676 & 18 \\
Chinese & 60 & 12 & 1341 & 28 \\
\hline
\end{tabular}

local extractors. Generally speaking, the extractors that are more confident with their predictions will result in less candidates, and also less constraints.

We also notice that, given thousands of different relations in the DBpedia or HudongBaike $\mathrm{KB}$, we only manage to map dozens of different relations from the corpora we used. In order to extract other types of relations, one may need to collect different resources in the future.

\subsection{Baselines and Competitors}

For a thorough examination, we compare with both traditional RE models and modern neural networks based RE models.

1. MaxEnt: Maximum Entropy [38] is a widely used baseline extractor in distantly supervised RE, which usually takes both lexical and syntactic features as input. In our experiments, we also incorporate $\mathrm{N}$-gram based lexical features for an expectation of higher recall.

2. MultiR: is a novel joint model that is designed to deal with the overlap between multiple relations [18].

3. MIML-RE: follows a two-step paradigm, which first uses a multi-class classifier to make latent predictions for the mentions, which are then fed to a bunch of binary classifiers to decide whether the entity pair holds the corresponding relation. MIML-RE is one of the state-of-the-art traditional feature based RE systems [19].

4. NN-avg: is a variant of the PCNN model in [20], where the embedding of an entity pair is obtained by averaging the embeddings of the sentences containing this pair. It is also used as a baseline extractor in [21]. 
5. NN-att: utilizes the attention mechanism to weight each sentence inside a bag ${ }^{6}$, while calculating the embedding for an entity pair [21]. NN-att is currently one of the state-of-the-art neural network RE algorithms.

Note that all the above models are originally designed to output entity pair level predictions.

Our general framework will take sentence level predictions as input, resolve the inconsistencies among them and finally output the entity pair level results. We thus collect the sentence level predictions from MaxEnt, MultiR, NN-avg and NN-at!? feed them to our framework, and compare our proposed method with their originally designed integration schemes as well as other existing integration methods.

1. Mintz++: is the baseline integration mechanism described in [19], which obtains multi-label outputs for an entity tuple by OR-ing all its local predictions. A similar approach is also adopted in MultiR.

2. NN-avg-bag: In neural network based models, one simple approach of integration is to average the embeddings of a bag's all sentences into one bag-level embedding, which is then used to obtain the bag-level predictions [21].

3. NN-att-bag: designs an attention mechanism to adaptively assign different weights for different sentences inside a bag, according to their importances for a candidate relation [21].

Following previous works [35], we use the Precision-Recall Curve (P-R Curve) as the evaluation criterion in our experiments. For each model, we rank all final predictions (in the entity pair level) in a descending order according to their confidence scores. In the ranked list, we compute precision/recall at each position, and then plot the P-R Curve for this model. Usually, the closer the curve is to the upper right corner, the better performance the model has.

\footnotetext{
${ }^{6} \mathrm{We}$ call all the sentences containing an entity pair as a bag for that entity pair.

${ }^{7} \mathrm{We}$ can not find local predictions from MIML-RE
} 


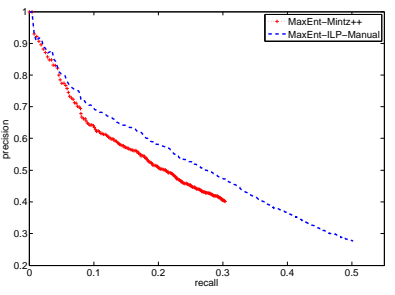

(a) The DBpedia Dataset

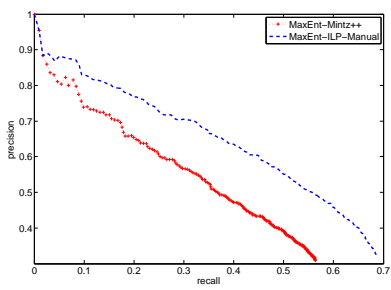

(b) The Chinese Dataset

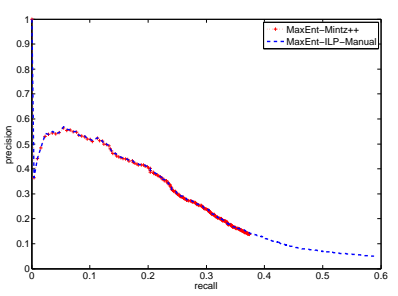

(c) The Riedel's Dataset

Figure 3: The results of the MaxEnt extractor on three datasets.

\subsection{Applying to Different Extractors}

Our main question is whether our proposed framework can handle the global inconsistencies among the local predictions from sentence-level relation extractors. We examine both traditional feature-based extractors (MaxEnt and MultiR) and neural networks based extractors ( $N N$-avg and $N N$-att) within our framework with hand crafted global clues. For each dataset we manually select around 60 relation pairs to capture the clues about argument types inconsistencies, and then include all the relations with unique argument values in $\mathcal{R}$. We first compare our framework with the Mintz++ style integration, which has been proved to be very competitive in [19].

The MaxEnt Extractor. Firstly, we feed both our framework and a Mintz++ style integrator with the MaxEnt's sentence-level predictions. As we can see in Figure 3, compared with the MaxEnt-Mintz++, our framework MaxEnt-ILP-Manual wins MaxEntMintz++ at almost every recall point, performing consistently better in both the DBpedia dataset and the Chinese dataset. Our ILP based framework takes the same sentencelevel local predictions as MaxEnt-Mintz++, but helps filter out incorrect predictions from the local MaxEnt extractor, leading to higher precisions.

However, in the Riedel's dataset, our framework cannot improve the performance. We manually investigate the dataset, and find that this dataset is dominated by three BIG relations:

/location/location/contains, /people/person/nationality and/people/person/place_lived, which cover about two-thirds of the entity tuples in total, and make the output of the local extractor bias even more to these BIG relations. Specifically, we find that about 
Table 2: Details of the improvements by our ILP framework in the DBpedia and Chinese datasets.

\begin{tabular}{lccc} 
Datasets & \# of eliminated & \# of corrected & \# of introduced w.r.t. Mintz++ \\
\hline DBpedia & 268 & 61 & 1426 \\
Chinese & 1506 & 14 & 283 \\
\hline
\end{tabular}

two-thirds of the local predictions are covered by one relation, /location/location/contains. These biased local predictions actually prevent us from collecting useful constraints from different kinds of clues. The reasons are twofold. Firstly, the type requirements of this relation are too general, e.g., it is not helpful at all to identify the difference between a city and a country since both of them can act as both subject and object for the relation/location/location/contains. Secondly, most entities are predicted to associate with only one relation, thus there will not be any disagreement among them, and we are not able to correct any wrong predictions, either. Other relations in this dataset may have some disagreements among local output, but they are too few to be captured by manually coded clues. Therefore, in this dataset, we cannot resolve the wrong local predictions by implicitly capturing the type requirements.

In order to better illustrate how the proposed framework actually works to improve performances, we compare the outputs of our framework and Mintz++, and investigate how many incorrect predictions are eliminated and corrected by our framework on the DBpedia and Chinese datasets. We also examine how many correct predictions are newly introduced by ILP, which are predicted as NA by the Mintz++ style integration.

The results summarized in Table 2 show that our framework can not only eliminate the incorrect predictions from local extractors, but also introduce more correct predictions, which are originally ranked the 2 nd and 3 rd in the local predictions, at the same time. We also find an interesting result: in the DBpedia dataset, our ILP framework is more likely to introduce correct predictions, while in the Chinese dataset, it tends to reduce more incorrect predictions. This agrees with what is shown in the P-R curves: in the DBpedia dataset, our framework extends longer along the recall axis, while in the Chinese dataset, it improves the precision significantly. It is also worth mentioning that the predictions being corrected are much less than the ones being eliminated or 


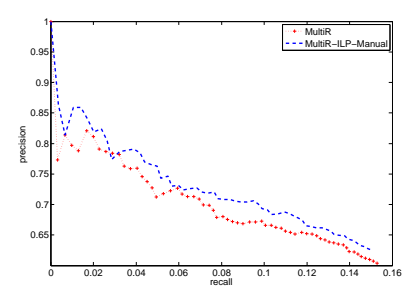

(a) The DBpedia Dataset

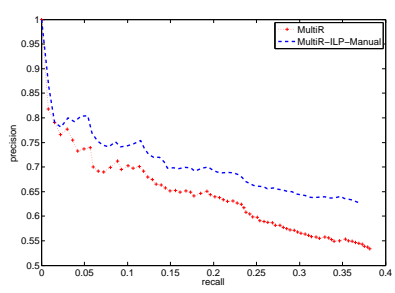

(b) The Chinese Dataset

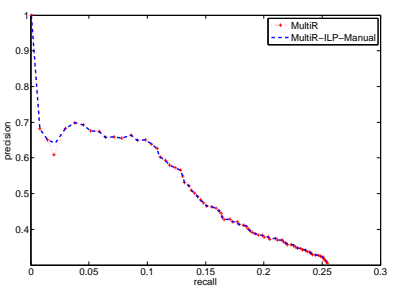

(c) The Riedel's Dataset

Figure 4: The results of the MultiR extractor on the three datasets.

newly introduced on both datasets. This is mainly because that when correcting a prediction, our framework should first identify an incorrect prediction for an entity pair, eliminate it, and pick out the next best prediction with a high enough confidence and mostly compatible with the constraints, at the same time.

The MultiR Extractor. MIML-RE and MultiR are two state-of-the-art models among traditional relation extractors. However, the MIML-RE model can only output entitypair level results, we thus fit MultiR's sentence-level extractor into our framework.

As we can see in Figure 4 , in both the DBpedia and Chinese datasets, our ILP optimized MultiR outperforms original MultiR in most parts of the curves. Note that, original MultiR adopts an integration scheme very similar to Mintz++ to merge their sentence-level predictions into entity-pair level. We think the reason is that our framework makes use of global clues to discard incorrect predictions, while the original MultiR does not.

In addition, the improvements by our framework are not as high as in the MaxEnt case, possibly due to the fact that the sentence-level MultiR does not perform well in these two datasets. Furthermore, the confidence scores output by MultiR are not normalized to the same scale, which makes it difficult in setting up a reasonable confidence threshold to select the candidate predictions and contributing to the objective function. As a result, we only use the top one result as the candidate, since including top two predictions without thresholding the confidences empirically leads to inferior performances, indicating that a probabilistic local extractor is more suitable for our framework. We also notice that our framework, again, does not bring significant im- 


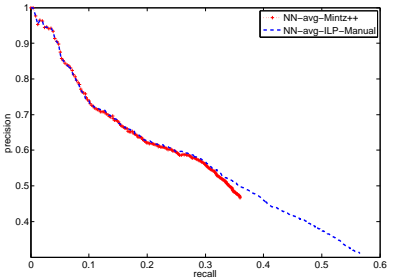

(a) The DBpedia Dataset



(d) The DBpedia Dataset

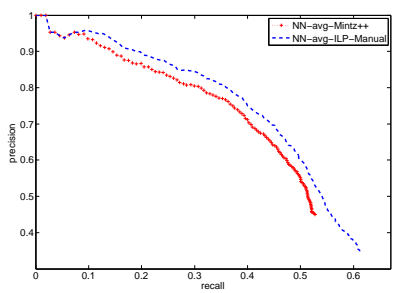

(b) The Chinese Dataset

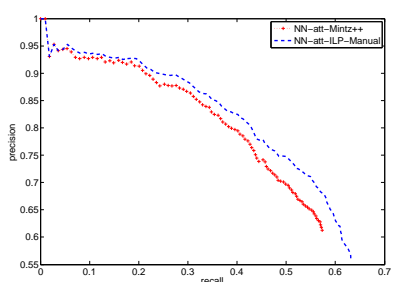

(e) The Chinese Dataset



(c) The Riedel's Dataset

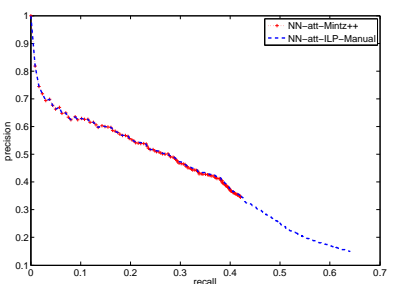

(f) The Riedel's Dataset

Figure 5: The results of ILP optimized NN models on the three datasets. (a)-(c) are results from $N N$-avg, while (d)-(f) are from $N N$-att.

provement in the Riedel's dataset, possibly due to the same reasons as discussed in previous section.

The Neural Network Extractors. Now we feed our framework with the sentence-level output from two neural network relation extractors, the average based model ( $N N$-avg) and the attention based (NN-att) [21].

From Figure 5 we can observe that, in the Chinese dataset, our ILP framework performs better than the Mintz++ style integrated NN extractors. However, in the DBpedia dataset, the NN improvement is not as significant as in the traditional models. One possible reason is that the result of $\mathrm{NN}$ extractors is considered as more accurate and confident than the traditional models, resulting in less constraints and less improvements accordingly. And again, there are still no improvements for both NN models in the Riedel's dataset.

From the above discussion, we find that our proposed framework can effectively work with different local relation extractors, both traditional feature based and neural network based, and improve the overall extraction performance when such global clues 


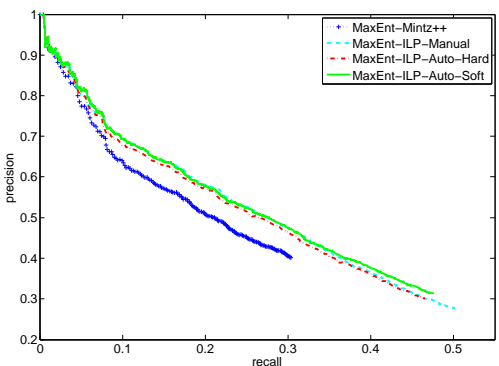

(a) The DBpedia Dataset

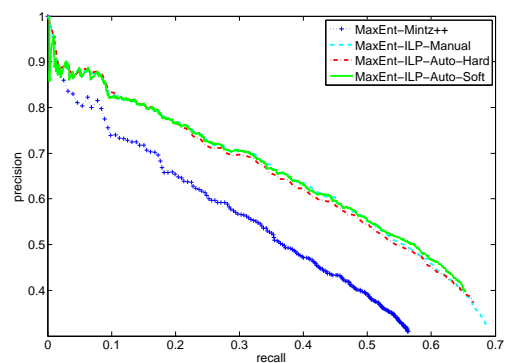

(b) The Chinese Dataset

Figure 6: Performance of automatic clues and manual clues with MaxEnt extractor.

are applicable to the datasets. And for the datasets where we fail to generate useful constraints, e.g., the Riedel's dataset, our framework does not hurt the performance.

\subsection{Automatically and Manually Obtained Clues}

Now we will investigate the difference between the manually obtained clues and automatically generated ones. The manually obtained clues can only be formulated into constraints in the hard style, while the automatically generated clues can be used in both hard style and soft style. Considering that the MultiR extractor does not perform as well as the MaxEnt and NN extractors, we only list the results of MaxEnt and NN extractors in the following.

Results on the MaxEnt Extractor. We first compare the performance of the manual clues in the hard style (Manual), automatic clues in the hard style (Auto-Hard) and automatic ones in the soft style (Auto-Soft) when they are applied to the output of the MaxEnt extractor.

In Figure 6, we can observe that the results with automatically obtained clues, no matter in hard or soft style, are comparable to those with manually refined clues. This indicates that the automatically collected clues can help to handle the inconsistencies among local predictions as manually refined clues do, and are shown to be effective in our framework.

We also find that the soft style formulation performs slightly better than the hard style on both datasets. We think the reason is that the automatically collected clues in 
a soft style formulation can take more unusual situations into consideration, while the hard style is well-directed according to the annotators.

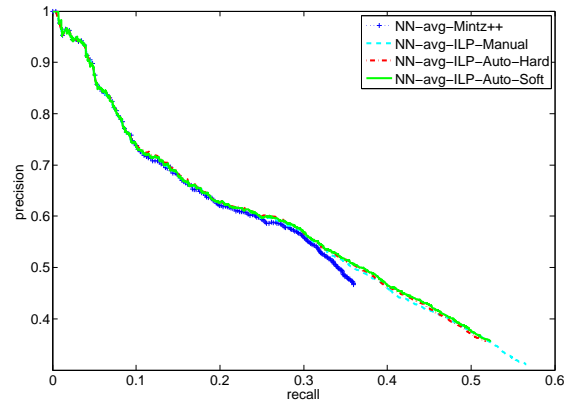

(a) The DBpedia Dataset

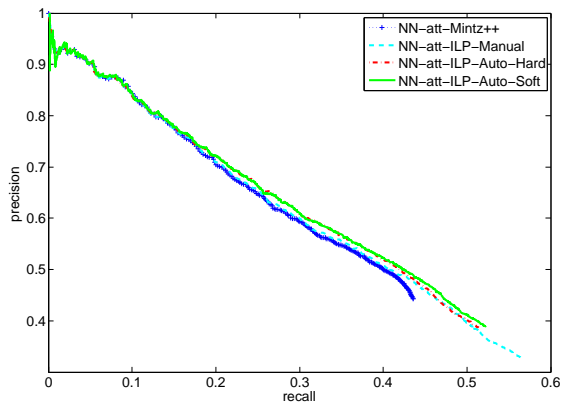

(c) The DBpedia Dataset

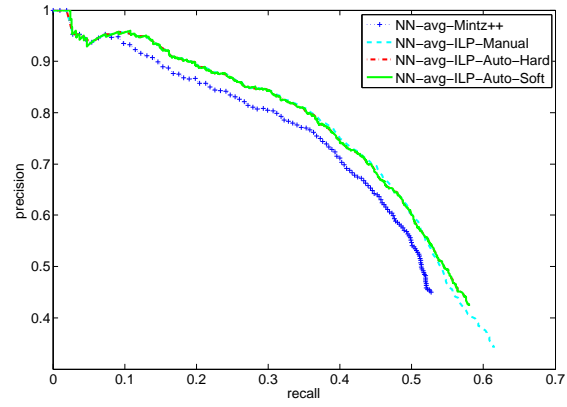

(b) The Chinese Dataset

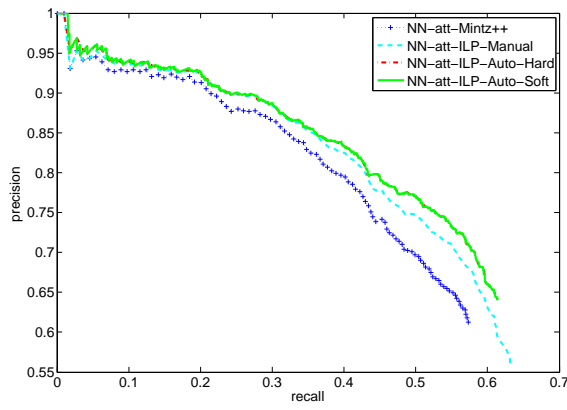

(d) The Chinese Dataset

Figure 7: Performance of automatic clues and manual clues with neural network based extractors.

Results on the Neural Network Extractors. As shown in Figure 7 in both datasets, the automatically collected clues can improve the NN models better than, or comparably to, the manually obtained ones. We also notice that in the Chinese dataset, the automatic clues can help $N N$-att to obtain more improvement against manual clues. After a careful check over the outputs, we find that in this dataset, most incorrect predictions in the output with manual clues belong to three relations, where, unfortunately, the manually obtained clues do not fully cover the clues about those relations, thus cannot help find enough disagreements to generate useful constraints. On the other hand, those involved relations are well covered by the automatically obtained clues. This points out the main shortcoming for manually collected clues that it requires more deep human 


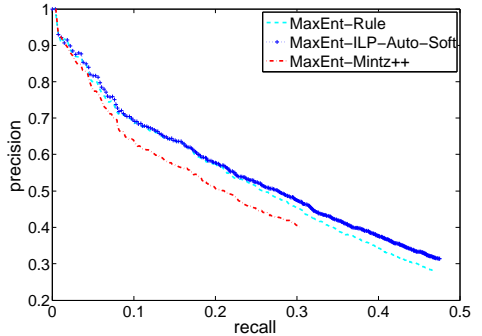

(a) The DBpedia Dataset



(b) The Chinese Dataset

Figure 8: Performance of the simple rule-based strategy and the ILP solver with the MaxEnt extractor.

involvement, and largely relies on the annotators' expertise.

By comparing the soft and hard style formulations with the automatic clues in Figure 7. we can see that both of them can effectively deal with the global inconsistencies among the output of $\mathrm{NN}$ extractors, and perform comparably to each other.

\subsection{Comparing ILP with A Simple Rule-based Solution}

As we can see in many ILP solutions, with the increasing number of entity pairs and candidate relations, the number of variables and constraints encoded for ILP will increase dramatically. As an alternative, one can also design a simple rule-based method to utilize our obtained clues. For instance, we can design the selection rule as: if two or more local predictions conflict with each other, we simply reserve the one with the highest confidence.

Results on the MaxEnt Extractor. We first compare this simple rule-based strategy with ILP using MaxEnt model as the local extractor. As we can see in Figure 8 , both the simple rule-based strategy and the ILP framework significantly improve over the baseline method in both datasets. And, in the DBpedia dataset, our ILP solution performs slightly better than the rule-based strategy when the recall is in the low region, and as the recall becomes higher, the improvement from the ILP solution becomes larger. And, surprisingly, on the Chinese dataset, the rule-based strategy achieves slightly higher precision in the low recall region, but when the recall becomes higher, the ILP solution obtains better performance again. 


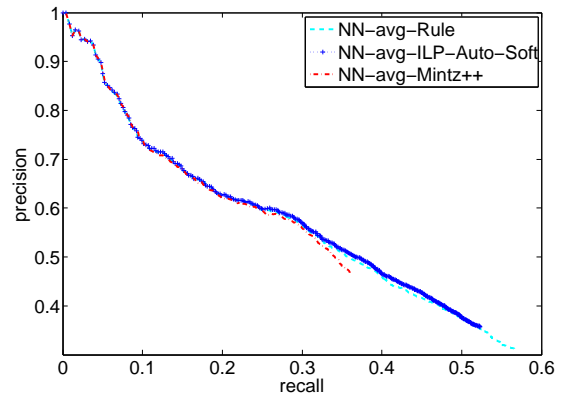

(a) The DBpedia Dataset

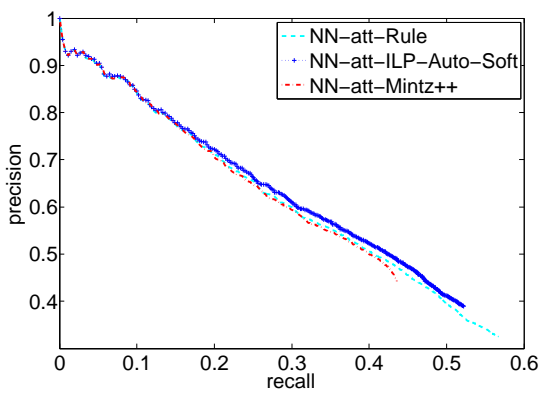

(c) The DBpedia Dataset

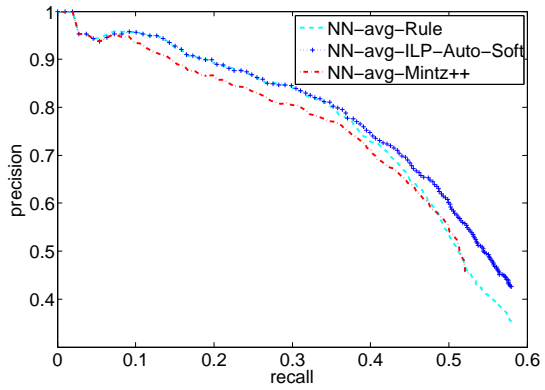

(b) The Chinese Dataset

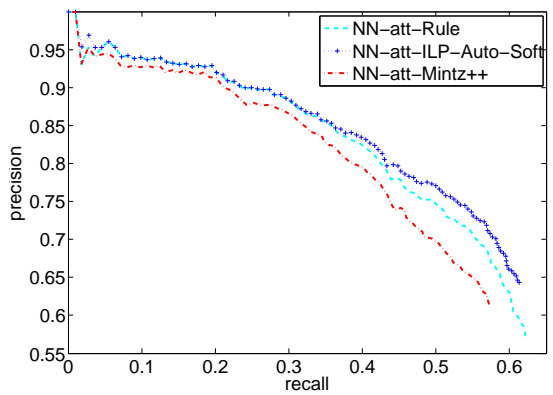

(d) The Chinese Dataset

Figure 9: Performance of the simple rule-based strategy and the ILP solver with the neural network based extractors.

Results on the NN Extractor. As is shown in Figure 9, when we use the NN models, both avg and att, as the preliminary extractor, on both datasets, our ILP based framework performs better than, or comparable to, the rule-based solution. All the results above indicate that compared with the simple heuristic technique, our ILP framework is more stable and works better in most situations, especially when the problem is complex and possibly hard to solve heuristically.

\subsection{Comparing with the State of the Arts}

In previous subsections, we have demonstrate the effectiveness of our framework with different local extractors and the global clues, collected either manually or automatically. Next, we compare our framework with both traditional and neural network state-of-the-art extraction models. Considering the fact that the automatic clues 


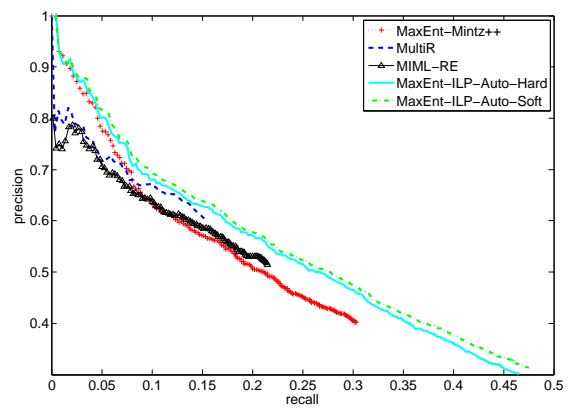

(a) The DBpedia Dataset

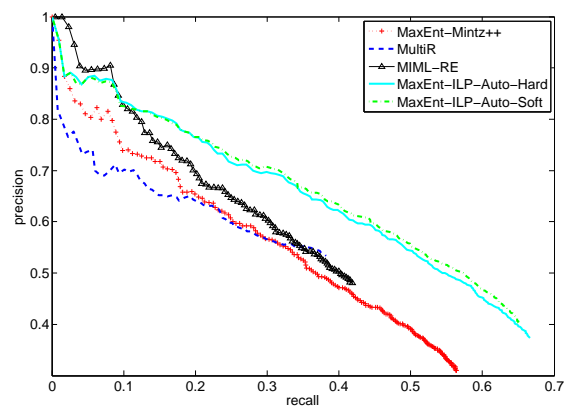

(b) The Chinese Dataset

Figure 10: Performance of our framework and traditional state-of-the-art extractors.

performs consistently better than or comparably to the manual ones, we will use the framework with automatically collected clues for detailed comparison and analysis.

Traditional Extractors. We first compare our ILP optimized MaxEnt with the traditional state-of-the-art extractors, MultiR and MIML-RE in Figure 10.

Compared with MultiR, our ILP framework obtains better results in both datasets. Especially in the Chinese dataset, the precision is improved around 10-16\% at the same recall points. Our proposed solution performs better compared to MIML-RE in the English dataset, and outperforms MIML-RE in the Chinese dataset, except in the lowrecall part $(<10 \%)$ of the P-R curve. This shows that encoding the relation background information into a simple MaxEnt model can outperform state-of-the-art traditional models.

Neural Network Extractors. Regarding the neural networks category, we build our solutions by optimizing two sentence-level NN extractors with our proposed ILP framework, and compare with the full state-of-the-art NN extractors [21].

As shown in Figure 11, in the Chinese dataset, our framework outperforms the original full models, NN-avg-bag and NN-att-bag, significantly on both averaging network or attention network, while in the DBpedia dataset, our framework performs comparably to the attention network, and slightly worse than the averaging network. The main difference between our solution and those two state-of-the-art models is that our 


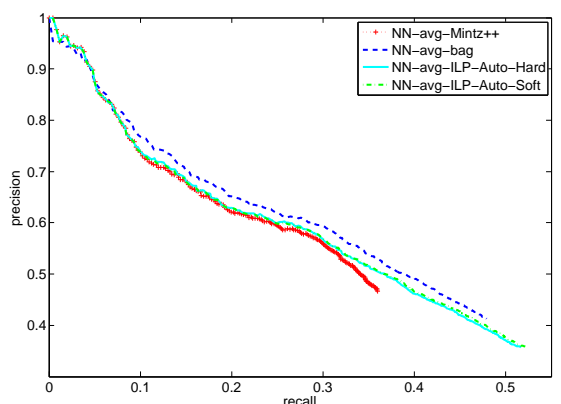

(a) The DBpedia Dataset

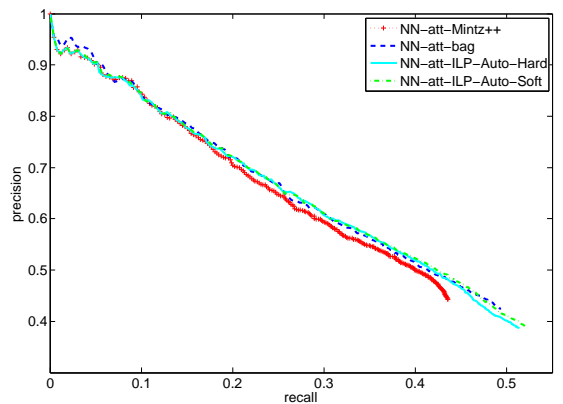

(c) The DBpedia Dataset

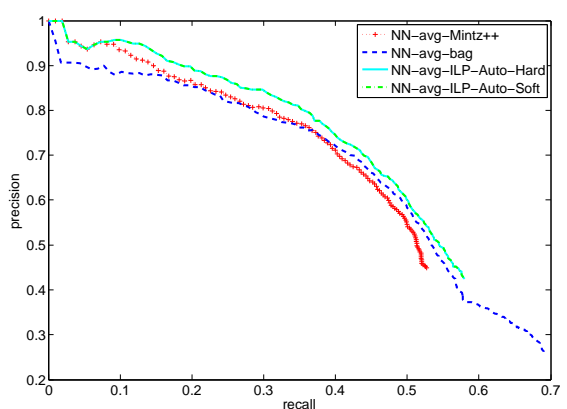

(b) The Chinese Dataset

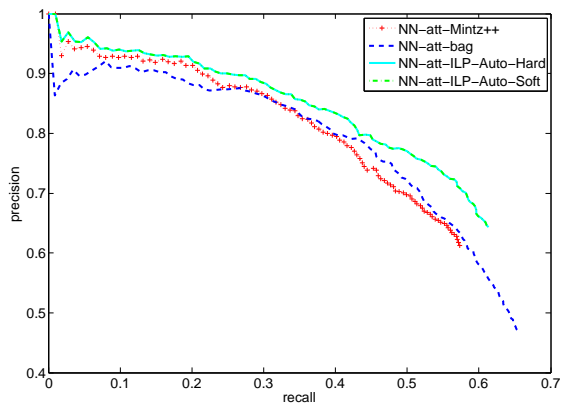

(d) The Chinese Dataset

Figure 11: Performance of our framework and the state-of-the-art neural networks based extractors.

framework focuses on exploring the global clues among different entity pairs and eliminating wrong predictions, while the state-of-the-art NN extractors focus on how to better merge different sentence-level mentions within an entity pair, e.g., averaging those mentions or attentively weighting them to represent the context between this entity pair. It would be an interesting direction to investigate how to combine their advantages in a unified framework, which we leave for future work.

\subsection{Impacts of Different Constraints}

Our ILP based framework exploits two kinds of constraints, regarding the argument type inconsistencies and violations of arguments' uniqueness, respectively, to address the global inconsistencies among local predictions, which are designed to implicitly encode relation requirements. We thus investigate the impact of those constraints used in the ILP framework. 
For the sake of clear and concise representation, we follow [19], to use the peak F1 score (highest F1 score) as the evaluation criterion.

The baseline, No-Constraint, is a variant of our framework without any constraints. It is different from Mintz++, since we use the approach in Section 3.1 to summarize the confidence scores of each relation and obtain the top 3 relations as the final results. It can help us to collect more potentially correct results. Note that No-Constraint has better or comparable F1 scores compared with Mintz++ in both datasets: in the DBpedia dataset Mintz++ is $34.7 \%$ and No-Constraint is $35.2 \%$, while in the Chinese dataset they are both $44.4 \%$. As shown in Table 3 , in the DBpedia dataset, the highest F1 score increases from $35.2 \%$ to $38.3 \%$ with the help of both kinds of clues, while in the Chinese dataset, all constraints contribute to an improvement of $8.4 \%$.

In the DBpedia dataset, the constraints with respect to the implicit argument type inconsistencies (SR+RO+RER) can improve the F1 score from $35.2 \%$ to $37.7 \%$, while in the Chinese dataset, the absolute improvement is as high as 5.0\%. The constraints targeting violations of arguments' uniqueness $(\mathrm{OU}+\mathrm{SU})$ can increase the F1 score by $1.5 \%$ and $5.1 \%$ on the DBpedia and Chinese dataset, respectively. Using constraints derived from only one kind of clues can also improve the performance, but not as well as using both of them.

We also examine how each sub-type of the constraints can improve the results. As shown in Table 3, the Sub-Rej (SR), Rel-Obj (RO) and Rel-Entity-Rel (RER) constraints (obtained from the $\mathcal{C}^{s r}, \mathcal{C}^{r o}$ and $\mathcal{C}^{r e r}$ clues in Section 3.4.3 can improve the F1 score by $1.1 \%, 1.6 \%, 1.2 \%$, respectively, in the DBpedia dataset, and $0.9 \%, 2.2 \%$ and $3.6 \%$, in the Chinese dataset. As for the two sub-types obtained according to arguments' uniqueness, the improvements of Obj-Unique (OU) and Subj-Unique (SU) (obtained from the $\mathcal{C}^{o u}$ and $\mathcal{C}^{s u}$ clues in Section 3.4.3) in the DBpedia and Chinese datasets are $1.3 \%$ and $0.6 \%, 5.2 \%$ and $0.0 \%$, respectively. From the results we can see that almost all sub-types of those constraints can contribute to the improvements on both datasets. Note that OU works better than SU, and SR leads to less improvement compared to RO and ERE. This is mainly because we have more OU constraints than SU, e.g., there are 1,372 OU constraints but only 358 SU constraints in the DBpedia dataset, and more RO/ERE constraints than SR, e.g., we generate about 10,000 SU 
Table 3: Results of different combinations of constraints on the DBpedia dataset and the Chinese dataset.

\begin{tabular}{c|ccc|ccc}
\hline \multirow{2}{*}{ Method } & \multicolumn{3}{|c|}{ DBpedia } & \multicolumn{3}{c}{ Chinese } \\
& P(\%) & $\mathbf{R}(\%)$ & $\mathbf{F 1}(\%)$ & $\mathbf{P}(\%)$ & $\mathbf{R}(\%)$ & F1(\%) \\
\hline No-Constraint & 34.1 & 36.3 & 35.2 & 43.3 & 45.7 & 44.4 \\
Subj-Rel $(S R)$ & 36.5 & 36.1 & 36.3 & 45.1 & 45.6 & 45.3 \\
Rel-Obj $(R O)$ & $\mathbf{3 7 . 0}$ & 36.6 & $\mathbf{3 6 . 8}$ & 48.0 & 45.4 & 46.6 \\
Rel-Entity-Rel $(R E R)$ & 35.4 & 37.5 & 36.4 & 46.8 & $\mathbf{4 9 . 3}$ & 48.0 \\
Obj-Unique $(O U)$ & 35.3 & $\mathbf{3 7 . 8}$ & 36.5 & $\mathbf{5 0 . 3}$ & 48.8 & $\mathbf{4 9 . 6}$ \\
Subj-Unique $(S U)$ & 35.3 & 36.3 & 35.8 & 43.3 & 45.6 & 44.4 \\
\hline \hline SR+RO+RER & $\mathbf{3 6 . 3}$ & $\mathbf{3 9 . 1}$ & $\mathbf{3 7 . 7}$ & 49.7 & $\mathbf{4 9 . 1}$ & 49.4 \\
OU+SU & 35.7 & 37.7 & 36.7 & $\mathbf{5 0 . 3}$ & 48.8 & $\mathbf{4 9 . 5}$ \\
\hline \hline All-Constraints & $\mathbf{3 7 . 5}$ & $\mathbf{3 9 . 1}$ & $\mathbf{3 8 . 3}$ & $\mathbf{5 2 . 8}$ & $\mathbf{5 2 . 9}$ & $\mathbf{5 2 . 8}$ \\
\hline
\end{tabular}

constraints, 24,000 RO constraints and 70,000 RER constraints in the DBpedia dataset. This indicates that more constraints may help discover more disagreements among local predictions, thus may yield better results.

\subsection{Other Factors}

Here, we discuss four main factors in our framework, i.e., the size of the clues, the number of candidate relations, the threshold for learning the clues, and the weight of the soft penalty, which will affect the effectiveness or efficiency of our method. For the sake of brevity, here we will take an ILP optimized MaxEnt model with automatically learnt soft-style constraints (MaxEnt-ILP-Auto-Soft) as an example, and analyze its performance on the DBpedia dataset.

The Size of Clues. We first investigate the impact of the size of clues. In the experiments, we add clues into the framework according to their related relations' proportions in the local predictions, and generate constraints based on those clues. For example, in the DBpedia dataset, the two biggest relations, Country and birthPlace, take up about $30 \%$ in the local predictions. We thus add the clues that are related to these two relations, and then move on to new clues related to other relations according to those 


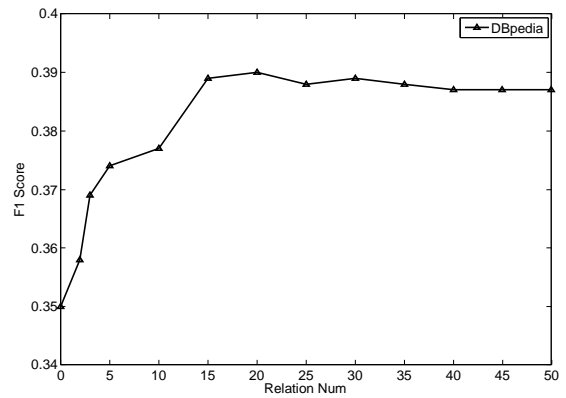

Figure 12: The performance of MaxEnt-ILP-Auto-Soft in the DBpedia dataset under different number of relations involved in the constraints. $\mathrm{X}$-axis is the number of relations, and Y-axis is the peak F1 score (highest F1 score).

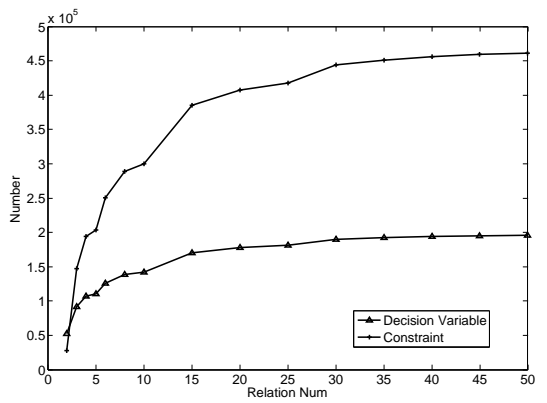

(a) Numbers of decision variables and constraints

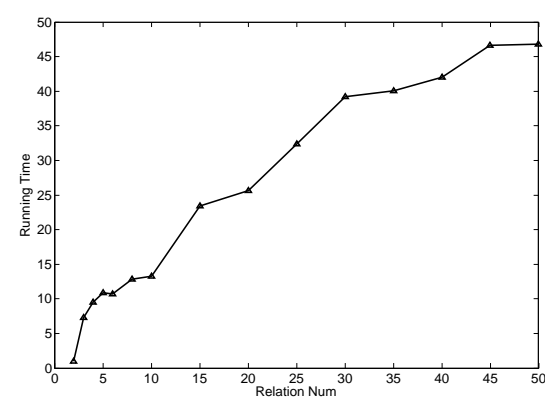

(b) The running time of the model

Figure 13: The efficiency of MaxEnt-ILP-Auto-Soft v.s. the number of the relations on the DBpedia dataset. In both (a) and (b), X-axis is the number of the relations. In (a), Y-axis is the number of the decision variables and the constraints, while in (b), Y-axis is the running time of our model measured in seconds.

relations' proportions.

As shown in Figure 14, the clues related to more local predictions would potentially solve more inconsistencies, thus are more effective. Adding the first two or three relations improves the performance significantly, and as more relations are added, the performances keep increasing until approaching the plateau.

A related efficiency issue is that when we introduce more clues, the numbers of the decision variables and the constraints, as well as the running time of the model would increase, as illustrated in Figure 13(a) and Figure 13(b). Specifically, the numbers of decision variables and constraints dramatically increase at first, and then with a slower 
growth. The reason is straightforward: we first add the relations with large proportions, which may bring more decision variables and constraints. As for the running time of the algorithm, along with more relations introduced, the model becomes more complex, and the time cost keeps increasing. Finally, the increase slows down and the running time almost stays still.

From the previous results we can observe that the running time of the optimization model is mostly affected by the numbers of the constraints and decision variables introduced to the model. More clues make the model perform better, but less efficient. In practice, users can trade off between the performance and the efficiency of our framework. For example, we can see from Figure 14 that the performance does not improve much after incorporating more than 20 relations, which means that we can prune the clues unrelated to the top 20 relations and maintain a reasonable performance.

We also notice that, due to the capacity of the ILP solver, our model cannot deal with too many relations at the same time. If there are too many relations involved, e.g., thousands of relations, there might be billions of constraints generated, and the running time will grow dramatically as well. One possible solution is to limit the maximum number of relations processed in one model. Then, how to properly split thousands of relations into several optimization models to achieve the globally best performance becomes an other interesting direction for future work.

The Number of Candidate Relations. In our framework, we select the top $n$ local predictions for each entity pair as the candidates for the following optimization procedure. As shown in Figure 14, we vary $n$ from 1 to 4 to show its impact to the extraction performance. We can observe that as $n$ increases from 1 to 3 , the precision of our framework does not improve much, but the recall increases dramatically. This indicates that selecting more candidates may help us collect more potentially correct predictions. However, obtaining 4 candidate relations can only bring a small improvement in recall compared to using 3 candidates. On the contrary, it makes the model more complex, since that will bring more constraints. To confirm, we investigate the local predictions in different datasets, and find that more than $80 \%$ of the correct predictions are among the top 3 . Thus, we finally select the top 3 predictions as the candidates in 


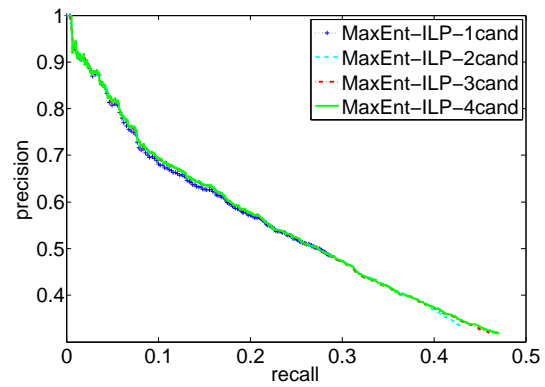

Figure 14: The performance of MaxEnt-ILP-Auto-Soft v.s. the number of candidate relations on the DBpedia dataset.



Figure 15: The performance of MaxEnt-ILP-Auto-Soft v.s. the threshold of learning the clues on the DBpedia dataset.

our framework.

The Threshold for Learning the Clues. As discussed in Section 3.3, when our model automatically collects the clues, it is crucial to set up a proper threshold $\kappa$ since it will determine the quality of the obtained constraints. Intuitively, as the threshold $\kappa$ gets larger, the number of collected clues will increase, but often lead to more constraints of lower quality. Figure 15 shows the performance of our framework as $\kappa$ varies from -1 to -4 (represented as MaxEnt-ILP-thre1, MaxEnt-ILP-thre2, MaxEnt-ILP-thre3 and MaxEnt-ILP-thre4). When comparing MaxEnt-ILP-thre1 and MaxEnt-ILP-thre2, we can see that their precisions are competitive within the low-recall region. After the recall is higher than 0.2, the precision of MaxEnt-ILP-thre2 is higher than MaxEntILP-thre1, and the final recall of MaxEnt-ILP-thre2 is also better than MaxEnt-ILP- 


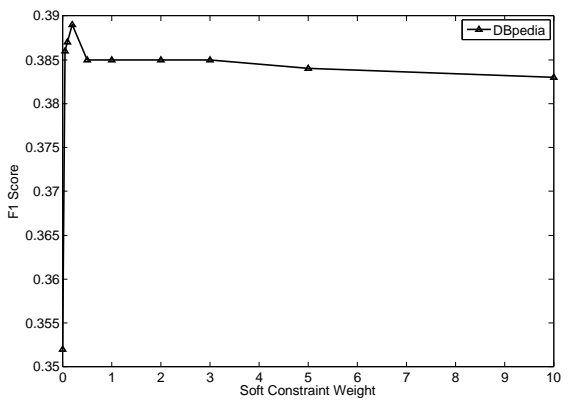

Figure 16: Performance of soft constraints v.s. the weight of the constraints. X-axis is the weight of the soft penalty, while Y-axis is the peak F1 score.

thre1. We think the reason may be that the low quality clues learnt from $\kappa=-1$ often hurt the correct predictions. The performance of MaxEnt-ILP-thre3 is better than both MaxEnt-ILP-thre1 and MaxEnt-ILP-thre2, indicating that $\kappa=-3$ can derive higher quality clues than -1 and -2 . As the threshold is set to -4 , the precision becomes lower almost at all recall points, since $\kappa=-4$ will lead to much fewer clues than MaxEntILP-thre3, thus can only eliminate fewer incorrect predictions.

The Weight for the Soft Penalty. There is a parameter $\alpha$ in the soft style formulation (Equation 9), which controls the importance of soft constraints. Intuitively, the larger $\alpha$ is, the more penalty we will obtain when we violate the constraints. When the penalty is large enough, it will be very expensive to violate a constraint, and we will not benefit anything from violating a constraint. That will make a constraint work as a hard one. Figure 16 shows how our framework performs as $\alpha$ increases from 0 to 10 .

We can see that as $\alpha$ increases from 0 , the performance first improves significantly, and then almost stays still. This indicates the performance of the soft constraints is not sensitive to the parameter $\alpha$.

\section{Conclusions and Future Work}

In this paper, we explore the global clues derived from structured knowledge bases to help resolve the disagreements among local relation predictions, thus reduce the incorrect predictions and improve the performance of relation extraction. Two kinds 
of clues, including implicit argument type information and argument cardinality information of relations are investigated. Those clues can be used to generate constraints in either hard style or soft style, both of which can be explored effectively in a constrained optimization framework, e.g., integer linear programming. Our framework outperforms the state-of-the-art models if we can find such clues in the knowledge base and they are applicable to the dataset. Furthermore, our framework is scalable for various local extractors, including traditional models and the modern neural network models. Additionally, we show that the clues can be learnt automatically from the KB, and lead to comparable or better performance to manually refined ones.

In the future, we will investigate how to incorporate new types of clues in our framework, and extend our framework to a large-scale relation extraction scenario. In terms of modeling, we would like to study a unified framework to combine the global clues among different entity pairs and the local information within an entity pair, which are currently treated in isolation.

\section{Acknowledgements}

We would like to thank Dong Wang and Kun Xu for their helpful discussions and comments. This work was supported by the National Hi-Tech R\&D Program of China [Grant Number 2015AA015403]; the National Science Foundation of China [Grant Numbers 61672057 and 61672058]; and the IBM Shared University Research Award.

\section{Appendix: Details about the DBpedia and Chinese Datasets}

\subsection{The DBpedia Dataset}

There are in total 51 relations used to construct our DBpedia dataset, as summarized in Table 4

As discussed in Section 3.2, we mainly examine two categories of clues, implicity argument types inconsistancies and violations of aruments' uniqueness. The former could be roughly divided into three subcategories, $\mathcal{C}^{s r}$, indicating a pair of relations

are inconsistent regarding their subjects, $\mathcal{C}^{r o}$, indicating the inconsistency regarding their objects, and $\mathcal{C}^{r e r}$ referring the inconsistency in terms of one relation's subject and 
Table 4: The 51 relations used to build the DBpedia dataset.

\begin{tabular}{ccccc}
\hline $\begin{array}{c}\text { region } \\
\text { child }\end{array}$ & residence & birthPlace & primeMinister & influenced \\
country & formerTeam & currentPartner & associatedBand & successor \\
keyPerson & knownFor & locationCountry & stateOfOrigin & product \\
location & coachedTeam & locatedInArea & foundationPerson & type \\
hometown & leaderName & nationality & subsidiary & owner \\
garrison & team & locationCity & foundationPlace & partner \\
occupation & vicePresident & regionServed & draftTeam & spouse \\
employer & debutTeam & parentCompany & headquarter & capital \\
state & president & owningCompany & influencedBy & city \\
associatedMusicalArtist & & & \\
\hline
\end{tabular}

the other one's object. The latter one, violations of aruments' uniqueness, focused on the argument cardinality requirements for a relation. Specifically, $\mathcal{C}^{o u}$ means a relation expects unique object, while $\mathcal{C}^{s u}$ indicates a relation requires unique subject for a given object.

We list the clues manually designed for the DBpedia dataset in Table 5

\subsection{The Chinese Dataset}

As summarized in Table 6, there are 28 relations used to construct our Chinese dataset. There are also two categories of clues manually annotated for the Chinese dataset, including 5 subcategories, as listed in Table 7 . 
Table 5: The manual clues designed for the DBpedia dataset.

$$
\mathcal{C}^{s r}
$$

$<$ country, capital $>$, < country, birthPlace $>$, < country, nationality $>$, < capital, deathPlace $>$, $<$ country, deathPlace $>,<$ capital, birthPlace $>,<$ country, residence $>,<$ team, location $>$, $<$ state, birthPlace $>,<$ state, deathPlace $>,<$ state, nationality $>,<$ leaderName, residence $>$, $<$ city, hometown $>$, < capital, birthPlace $>,<$ capital, deathPlace $>,<$ capital, state $>$ $<$ team, associatedMusicalArtist $>,<$ country, hometown $>$

\begin{tabular}{c}
\hline $\mathcal{C}^{r o}$ \\
\hline country, capital $>,<$ country, state $>,<$ country, team $>,<$ country, city $>$, \\
$<$ country, state $>,<$ city, state $>,<$ nationality, capital $>,<$ nationality, state $>$, \\
$<$ nationality, city $>,<$ city, team $>,<$ leaderName, team $>,<$ location, team $>$, \\
$<$ state, team $>,<$ almaMater, nationality $>,<$ almaMater, city $>,<$ almaMater, country $>$, \\
$<$ state, locationCity $>,<$ locationCountry, locationCity $>,<$ locationCountry, state $>$, \\
$<$ nationality, locationCity $>,<$ locationCountry, city $>$ \\
$\mathcal{C}^{r e r}$
\end{tabular}

$<$ country, country $>,<$ capital, capital $>,<$ team, team $>,<$ country, birthPlace $>$, $<$ city, birthPlace $>$, < region, birthPlace $>,<$ country, deathPlace $>,<$ city, deathPlace $>$, $<$ country, nationality $>$, <nationality, country $>$, $<$ city, region $>,<$ country, city $>$, $<$ almaMater, nationality $>,<$ country, state $>,<$ city, capital $>,<$ nationality, country $>$, $<$ locationCountry, country $>,<$ country, residence $>,<$ leaderName, team $>$, $<$ city, residence $>,<$ president, team $>,<$ team, nationality $>,<$ team, debutTeam $>$ $\mathcal{C}^{\text {ou }}$ nationality, country, capital, state, stateOfOrigin, locationCountry $\mathcal{C}^{s u}$ capital, subsidiary 
Table 6: The 28 relations used to construct the Chinese dataset.

\begin{tabular}{cccccc}
\hline 所属洲 & 国籍 & 籍贯 & 著名景点 & 首都 & 所属地区 \\
下辖地区 & 名人 & 法人 & 毕业院校 & 创建地点 & 总部所在地 \\
生产厂商 & 执政党 & 政党 & 现任领导人 & 校长 & 导演 \\
知名企业 & 作者 & 出生地 & 知名校友 & 主场 & 代表队员 \\
主演 & 主编 & 品牌 & 上市市场 & & \\
\hline
\end{tabular}

Table 7: The manual clues designed for the Chinese dataset.

\section{$\mathcal{C}^{s r}$}

$<$ 国籍, 下辖地区 $>,<$ 国籍, 所属洲 $>,<$ 国籍, 首都 $>,<$ 国籍, 现任领导人 $>$,

$<$ 国籍, 下辖地区 $>$, $<$ 国籍, 首都 $>,<$ 首都, 籍贯 $>,<$ 所属洲, 籍贯 $>$,

$<$ 著名景点, 国籍 $>,<$ 下辖地区, 国籍 $>,<$ 所属地区, 国籍 $>,<$ 著名景点, 籍贯 $>$,

$<$ 籍贯, 所属洲 $>,<$ 籍贯, 代表队员 $>,<$ 籍贯, 执政党 $>,<$ 籍贯, 所属地区 $>$,

$<$ 国籍, 执政党 $>,<$ 国籍, 创建地点 $>,<$ 所属洲, 创建地点 $>,<$ 著名景点, 创建地点 $>$

\section{$\mathcal{C}^{\text {ro }}$}

<国籍, 所属洲 $>,<$ 国籍, 毕业院校 $>,<$ 国籍, 首都 $>,<$ 国籍, 现任领导人 $>$,

<国籍, 所属洲 >, <著名景点, 所属洲 >, <首都, 所属洲 >, <籍贯, 毕业院校 $>$,

<籍贯, 所属洲 $>,<$ 首都, 毕业院校 $>,<$ 执政党, 现任领导人 >, <校长, 主场 $>$,

$<$ 品牌, 主演 $>,<$ 上市市场, 主演 $>,<$ 校长, 导演 $>,<$ 代表队员, 校长 $>$,

$<$ 代表队员, 导演 $>,<$ 主演, 校长 $>,<$ 生产厂商, 执政党 $>,<$ 政党, 首都 $>$

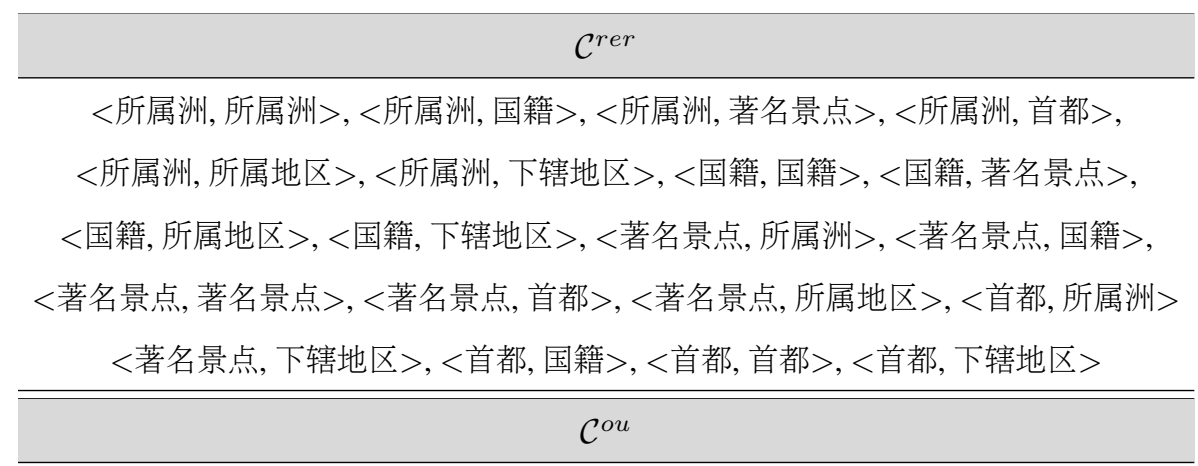

所属洲, 首都, 生产厂商, 主场, 政党, 创建地点, 国籍, 籍贯

$$
\mathcal{C}^{s u}
$$

首都, 执政党, 现任领导人, 品牌 


\section{References}

\section{References}

[1] F. Suchanek, J. Fan, R. Hoffmann, S. Riedel, P. P. Talukdar, Advances in automated knowledge base construction, in: SIGMOD Records Journal, 2013.

[2] L. Yao, S. Riedel, A. McCallum, Collective cross-document relation extraction without labelled data, in: Proceedings of the Conference on Empirical Methods in Natural Language Processing, EMNLP '10, Association for Computational Linguistics, Stroudsburg, PA, USA, 2010, pp. 1013-1023.

[3] X. Zhang, J. Zhang, J. Zeng, J. Yan, Z. Chen, Z. Sui, Towards accurate distant supervision for relational facts extraction, in: Proceedings of the 51st Annual Meeting of the Association for Computational Linguistics (Volume 2: Short Papers), Association for Computational Linguistics, Sofia, Bulgaria, 2013, pp. 810-815. URL http://www.aclweb.org/anthology/P13-2141

[4] M. Koch, J. Gilmer, S. Soderland, D. S. Weld, Type-aware distantly supervised relation extraction with linked arguments in: Proceedings of the 2014 Conference on Empirical Methods in Natural Language Processing (EMNLP), Association for Computational Linguistics, Doha, Qatar, 2014, pp. 1891-1901. URL http://www.aclweb.org/anthology/D14-1203

[5] H. Ji, R. Grishman, H. Dang, Overview of the tac2011 knowledge base population track, in: Proceedings of the Text Analysis Conference, 2011.

[6] S. Soderland, D. Fisher, J. Aseltine, W. Lehnert, Crystal: Inducing a conceptual dictionary, in: Proceedings of the 14th International Joint Conference on Artificial Intelligence - Volume 2, IJCAI'95, Morgan Kaufmann Publishers Inc., San Francisco, CA, USA, 1995, pp. 1314-1319.

[7] S. Zhao, R. Grishman, Extracting relations with integrated information using kernel methods, in: Proceedings of the 43rd Annual Meeting on Association for Computational Linguistics, ACL '05, Association for Computational Linguistics, Stroudsburg, PA, USA, 2005, pp. 419-426. 
[8] Y. Chen, Q. Zheng, P. Chen, Feature assembly method for extracting relations in chinese, Artificial Intelligence 228 (2015) 179-194.

[9] M. Banko, M. J. Cafarella, S. Soderland, M. Broadhead, O. Etzioni, Open information extraction from the web, in: Proceedings of International Joint Conference on Artificial Intelligence, IJCAI'07, 2007, pp. 2670-2676.

[10] A. Fader, S. Soderland, O. Etzioni, Identifying relations for open information extraction, in: Proceedings of the Conference on Empirical Methods in Natural Language Processing, EMNLP '11, Association for Computational Linguistics, Stroudsburg, PA, USA, 2011, pp. 1535-1545.

[11] F. Wu, D. S. Weld, Open information extraction using wikipedia, in: Proceedings of the Annual Meeting of the Association for Computational Linguistics, ACL '10, Association for Computational Linguistics, Stroudsburg, PA, USA, 2010, pp. 118-127.

[12] L. Qiu, Y. Zhang, Zore: A syntax-based system for chinese open relation extraction, in: Proceedings of the 2014 Conference on Empirical Methods in Natural Language Processing (EMNLP), Association for Computational Linguistics, Doha, Qatar, 2014, pp. 1870-1880.

URL http://www.aclweb.org/anthology/D14-1201

[13] Y. Xu, M.-Y. Kim, K. Quinn, R. Goebel, D. Barbosa, Open information extraction with tree kernels, in: Proceedings of the 2013 Conference of the North American Chapter of the Association for Computational Linguistics: Human Language Technologies, Association for Computational Linguistics, Atlanta, Georgia, 2013, pp. 868-877.

URL http://www.aclweb.org/anthology/N13-1107

[14] A. Carlson, J. Betteridge, B. Kisiel, B. Settles, E. H. Jr., T. Mitchell, Toward an architecture for never-ending language learning, in: Proceedings of the Conference on Artificial Intelligence (AAAI), AAAI Press, 2010, pp. 1306-1313. 
[15] R. C. Bunescu, Learning to extract relations from the web using minimal supervision, in: Proceedings of the 45th Annual Meeting of the Association for Computational Linguistics (ACL07), 2007.

[16] M. Mintz, S. Bills, R. Snow, D. Jurafsky, Distant supervision for relation extraction without labeled data, in: Proceedings of the Joint Conference of the 47th Annual Meeting of the ACL and the 4th IJCNLP of the AFNLP: Volume 2 Volume 2, ACL '09, 2009, pp. 1003-1011.

[17] M. Surdeanu, D. McClosky, J. Tibshirani, J. Bauer, A. X. Chang, V. I. Spitkovsky, C. D. Manning, A simple distant supervision approach for the TAC-KBP slot filling task, in: Proceedings of the Third Text Analysis Conference (TAC 2010), Gaithersburg, Maryland, USA, 2010.

[18] R. Hoffmann, C. Zhang, X. Ling, L. Zettlemoyer, D. S. Weld, Knowledge-based weak supervision for information extraction of overlapping relations, in: Proceedings of the 49th Annual Meeting of the Association for Computational Linguistics: Human Language Technologies, Association for Computational Linguistics, Portland, Oregon, USA, 2011, pp. 541-550.

URL http://www.aclweb.org/anthology/P11-1055

[19] M. Surdeanu, J. Tibshirani, R. Nallapati, C. D. Manning, Multi-instance multilabel learning for relation extraction., in: Proceedings of the 2012 Joint Conference on Empirical Methods in Natural Language Processing and Computational Natural Language Learning, Association for Computational Linguistics, 2012, pp. 455-465.

[20] D. Zeng, K. Liu, Y. Chen, J. Zhao, Distant supervision for relation extraction via piecewise convolutional neural networks, in: Proceedings of the 2015 Conference on Empirical Methods in Natural Language Processing, Association for Computational Linguistics, Lisbon, Portugal, 2015, pp. 1753-1762.

URL http://aclweb.org/anthology/D15-1203

[21] Y. Lin, S. Shen, Z. Liu, H. Luan, M. Sun, Neural relation extraction with selective 
attention over instances, in: Proceedings of the 54th Annual Meeting of the Association for Computational Linguistics (Volume 1: Long Papers), Association for Computational Linguistics, Berlin, Germany, 2016, pp. 2124-2133.

URL http://www.aclweb.org/anthology/P16-1200

[22] S. Takamatsu, I. Sato, H. Nakagawa, Reducing wrong labels in distant supervision for relation extraction, in: Proceedings of the 50th Annual Meeting of the Association for Computational Linguistics: Long Papers - Volume 1, ACL '12, Association for Computational Linguistics, Stroudsburg, PA, USA, 2012, pp. $721-729$.

[23] W. Xu, R. Hoffmann, L. Zhao, R. Grishman, Filling knowledge base gaps for distant supervision of relation extraction, in: Proceedings of the 51st Annual Meeting of the Association for Computational Linguistics (Volume 2: Short Papers), Association for Computational Linguistics, Sofia, Bulgaria, 2013, pp. 665-670.

URL http://www.aclweb.org/anthology/P13-2117

[24] M. Pershina, B. Min, W. Xu, R. Grishman, Infusion of labeled data into distant supervision for relation extraction, in: Proceedings of the 52nd Annual Meeting of the Association for Computational Linguistics (Volume 2: Short Papers), Association for Computational Linguistics, Baltimore, Maryland, 2014, pp. 732-738. URL http://www.aclweb.org/anthology/P14-2119

[25] G. Angeli, J. Tibshirani, J. Wu, C. D. Manning, Combining distant and partial supervision for relation extraction, in: Proceedings of the 2014 Conference on Empirical Methods in Natural Language Processing (EMNLP), Association for Computational Linguistics, Doha, Qatar, 2014, pp. 1556-1567.

URL http://www.aclweb.org/anthology/D14-1164

[26] C. Wang, J. Fan, A. Kalyanpur, D. Gondek, Relation extraction with relation topics, in: Proceedings of the 2011 Conference on Empirical Methods in Natural Language Processing, Association for Computational Linguistics, Edinburgh, Scotland, UK., 2011, pp. 1426-1436. 
[27] S. Riedel, L. Yao, B. M. Marlin, A. McCallum, Relation extraction with matrix factorization and universal schemas, in: Joint Human Language Technology Conference/Annual Meeting of the North American Chapter of the Association for Computational Linguistics (HLT-NAACL '13), 2013.

[28] H. Ye, W. Chao, Z. Luo, Z. Li, Jointly extracting relations with class ties via effective deep ranking, in: Proceedings of the 55th Annual Meeting of the Association for Computational Linguistics (Volume 1: Long Papers), Association for Computational Linguistics, Vancouver, Canada, 2017, pp. 1810-1820.

[29] D. Krompass, S. Baier, V. Tresp, Type-constrained representation learning in knowledge graphs., in: International Semantic Web Conference (1), Vol. 9366 of Lecture Notes in Computer Science, Springer, 2015, pp. 640-655.

[30] R. Xie, Z. Liu, J. Jia, H. Luan, M. Sun, Representation learning of knowledge graphs with entity descriptions, in: Proceedings of the Thirtieth AAAI Conference on Artificial Intelligence, February 12-17, 2016, Phoenix, Arizona, USA., 2016, pp. 2659-2665.

[31] Q. Li, S. Anzaroot, W.-P. Lin, X. Li, H. Ji, Joint inference for cross-document information extraction, in: Proceedings of the 20th ACM International Conference on Information and Knowledge Management, CIKM '11, ACM, New York, NY, USA, 2011, pp. 2225-2228.

[32] Q. Li, H. Ji, L. Huang, Joint event extraction via structured prediction with global features., in: Proceedings of the Annual Meeting of the Association for Computational Linguistics, The Association for Computer Linguistics, 2013, pp. 73-82.

[33] O. L. de Lacalle, M. Lapata, Unsupervised relation extraction with general domain knowledge., in: Proceedings of the Conference on Empirical Methods in Natural Language Processing, Association for Computational Linguistics, 2013, pp. 415-425.

[34] S. Kulczynski, Die pflanzenassoziationen der pieninen, Bulletin International de 
l'Academie Polonaise des Sciences et des Lettres, Classe des Sciences Mathematiques et Naturelles, B (Sciences Naturelles) Suppl. II (1927) 57-203.

[35] S. Riedel, L. Yao, A. McCallum, Modeling relations and their mentions without labeled text, in: Machine Learning and Knowledge Discovery in Databases, Vol. 6323 of Lecture Notes in Computer Science, Springer Berlin / Heidelberg, 2010, pp. 148-163.

[36] E. Sandhaus, The new york times annotated corpus ldc2008t19, in: Linguistic Data Consortium, Philadelphia, 2008.

[37] C. Bizer, J. Lehmann, G. Kobilarov, S. Auer, C. Becker, R. Cyganiak, S. Hellmann, Dbpedia - a crystallization point for the web of data, Web Semantics 7 (2009) 154-165.

[38] A. L. Berger, V. J. D. Pietra, S. A. D. Pietra, A maximum entropy approach to natural language processing, Computational Linguistics 22 (1) (1996) 39-71. 